\title{
GROUP EXTENSIONS AND COHOMOLOGY FOR LOCALLY COMPACT GROUPS. III
}

\author{
BY
}

\author{
CALVIN C. MOORE $\left({ }^{1}\right)$
}

\begin{abstract}
We shall define and develop the properties of cohomology groups $H^{n}(G, A)$ which can be associated to a pair $(G, A)$ where $G$ is a separable locally compact group operating as a topological transformation group of automorphisms on the polonais abelian group $A$. This work extends the results in [29] and [30], and these groups are to be viewed as analogues of the EilenbergMac Lane groups for discrete $G$ and $A$. Our cohomology groups in dimension one are classes of continuous crossed homomorphisms, and in dimension two classify topological group extensions of $G$ by $A$. We characterize our cohomology groups in all dimensions axiomatically, and show that two different cochain complexes can be used to construct them. We define induced modules and prove a version of Shapiro's lemma which includes as a special case the Mackey imprimitivity theorem. We show that the abelian groups $H^{n}(G, A)$ are themselves topological groups in a natural way and we investigate this additional structure.
\end{abstract}

1. In two previous papers [29], [30], we studied group extensions of locally compact groups, and introduced a cohomology theory analogous to the Eilenberg-Mac Lane theory for abstract groups which was appropriate for the study of such topological group extensions. More precisely, we considered a locally compact separable (i.e. second countable) group $G$ which operates as a topological transformation group of automorphisms on a locally compact abelian group $\boldsymbol{A}$ in which case one says that $\boldsymbol{A}$ is a (topological) $\boldsymbol{G}$-module, and we introduced cohomology groups $H^{n}(G, A), n \geqslant 0$, defined for such a pair and having the usual functoral properties. For $n=0, H^{0}(G, A)=A^{G}$, the $G$-fixed points in $A$, and for $n=1, H^{1}(G, A)$ is the group of all continuous crossed homomorphisms of $G$ into the $G$-module $A$, modulo the principal ones, and finally for $n=2, H^{2}(G, A)$ is the group of equivalence classes of topological group extensions of $G$ by the $G$-module $A$. (In this $A$ is the normal subgroup of the extension, and $G$ is the quotient group; for a precise definition of this notion, see [29] or below in §2.)

These cohomology groups, while very useful, have several defects; the first of these is that there do not appear to be any cohomologically trivial modules,

Received by the editors April 5, 1975.

AMS (MOS) subject classifications (1970). Primary 22 D05.

(1) Research supported in part by National Science Foundation Grant GP-30798X. 
that is modules such that $H^{n}(G, A)=0$ for $n \geqslant 1$. As a result, one is apparently deprived of the technique of dimension shifting, and hence precluded from employing systematically the standard techniques of homological algebra, and in particular one has next to zero information about the higher dimensional groups. A second and related difficulty is that when we construct resolutions of the module $A$ to be used in defining the cohomology groups, the groups appearing in this resolution are not locally compact, and indeed do not even admit a natural topology; this is unsatisfactory both aesthetically and technically. A third defect is that one would hope to have cohomology groups $H^{n}(G, A)$ which are themselves topological groups since one starts with topological data, namely $G$ and $A$. For $n=1$, there is a reasonable topology, and for $n=2$, we were able to topologize $H^{2}(G, A)$ by rather ad hoc techniques in some cases in [30]. Finally the restriction that $A$ be locally compact excludes from systematic treatment many interesting and useful examples, see for instance [13], [14], [26]. In this paper we shall introduce modifications of the original treatment in [29] and [30] which overcome all of these defects at once. The first modification is to extend the category of $G$-modules to include all so called polonais $G$-modules instead of just the locally compact ones: In this larger category we find many cohomologically trivial modules so the technique of dimension shifting is available. We shall also be able to present a short list of axioms which characterize our cohomology groups, and finally this wider category of $G$-modules will include many new examples of considerable interest. We are also able to construct induced modules in this larger category and establish a version of Shapiro's lemma relating the cohomology of a module to the cohomology of the induced module. As a special case of this we obtain Mackey's imprimitivity theorem [22].

This larger category of polonais $G$-modules could have been treated before except that there was no proof that $H^{2}(G, A)$ could be identified as the classes of group extensions of $G$ by $A$. We give a proof of this for polonais $A$ which in many ways is simpler than Mackey's original proof in [24] for locally compact $A$. Furthermore we shall construct a new resolution of $A$ within the category of polonais groups and so in particular the cohomology group will come automatically equipped with the structure of topological group. In our treatment $G$ will continue to be locally compact, and although there is some asymmetry here, we do pick up most of the important applications. Some results are known for polonais $G$ [4], but at the moment we lack a systematic treatment of this case.

There is one final difficulty in considering the cohomology of topological groups which to some extent is incurable, and this is the fact that the image of a continuous map of one topological group into another need not have a closed range, and so the map does not have a reasonable cokernel. This is a fact of life and we must live with it; to partially deal with it we shall (hesitantly) introduce 
the notion of pseudo-polonais $G$-module, but we shall not, however, pursue this, mostly because it does not add that much to the range of applicability of our theory. We note that many of our results were announced without proof in [31, Part II].

2. We shall begin with some preliminaries about the modules to be considered. Let $G$ be a second countable topological group which we always assume to be Hausdorff unless explicitly stated otherwise. Then $G$ admits three natural uniformities, the left uniformity, the right uniformity, and the two sided uniformity $U$, which is defined as the smallest uniformity containing the previous two.

Definition. Such a group $G$ will be said to be polonais (or $G \in P^{\prime}$ ) if $U$ is a complete conformity.

Proposition 1. A topological group is polonais if and only if its topology admits a separable complete metric $\rho$. Moreover if $G$ is polonais, and if $\rho_{1}$ is a left invariant metric, and $\rho_{2}$ a right invariant metric for $G$ (which always exist), then $\rho=\rho_{1}+\rho_{2}$ is complete.

Proof. This is in $[17$, p. 211].

This proposition says that a second countable topological group is polonais if and only if the underlying topological space of $G$ is polonais in the sense of [2], hence our terminology. We note that any second countable topological group may be embedded as a dense subgroup of a polonais group-just complete the group relative to its two-sided uniformity [17, p. 211]. Moreover, any separable (i.e. second countable) locally compact group is polonais, since in this case both the left and the right uniformities, and hence also $U$, are complete. Any separable Banach space or more generally any separable Fréchet space is polonais. Finally, and this is one of our most important examples, the group $U(H)$ of unitary operators on a separable Hilbert space $H$ is polonais for the strong (or equivalently the weak) operator topology (see [8]).

We now list several constructions which preserve this property of completeness.

Proposition 2. Let $G_{i}, i=1,2, \ldots$, be a sequence of polonais groups; then $\Pi G_{i}$, the Cartesian product, with the product topology is polonais.

Proof. This is immediate.

Let us consider a sequence of three topological groups with homomorphisms $i$ and $j$ :

$$
0 \rightarrow G^{\prime} \stackrel{i}{\rightarrow} G \stackrel{j}{\rightarrow} G^{\prime \prime} \rightarrow 0
$$

Definition. The sequence above is a short exact sequence of topological groups if it is algebraically exact (that is, $i$ is injective, $j$ is surjective, and $\operatorname{kernel}(j)$ 
= image $(i)$ ), and if $i$ is a homeomorphism onto its image, and if $j$ is continuous and open.

Note that it is not enough to assume that $i$ and $j$ are continuous, for then $G^{\prime}$ viewed as a subgroup may not have the relative topology, and the natural group isomorphism $j^{\prime}$ of $G$ /image $(i)$ onto $G^{\prime \prime}$ may not be a homeomorphism. We have the following fact which we will not prove here in complete generality-that is due to L. Brown. We prove the result in sufficient generality for our purposes.

Proposition 3. Consider a short exact sequence of Hausdorff topological groups $0 \rightarrow G^{\prime} \rightarrow G \rightarrow G^{\prime \prime} \rightarrow 0$. Then $G$ is polonais if and only if $G^{\prime}$ and $G^{\prime \prime}$ are.

Proof. If $G$ is polonais, then as $G^{\prime \prime}$ is Hausdorff, exactness implies that $i\left(G^{\prime}\right)$ is closed, hence polonais in the relative topology, and hence that $G^{\prime}$ is polonais. To show that $G^{\prime \prime}$ is polonais, we have to show that quotient group of a polonais group is polonais. This was established in general by L. Brown [6] and is nontrivial. We shall only really need the result when $G$ is abelian, and here it is very easy. Indeed let $\rho$ be an invariant complete metric on $G$, and define a metric $\bar{\rho}$ on $G / H$ ( $H$ any closed subgroup) by $\bar{\rho}(x H, y H)=\rho(x H, y H)$, the distance between these two cosets in $G$. It may be verified that $\bar{\rho}$ is complete and does the trick.

Conversely suppose that $G^{\prime}$ and $G^{\prime \prime}$ are complete; let us denote by $\bar{G}$ the completion of $G$ relative to its two sided uniformity so that $\bar{G}$ is complete. Then $j$ as a map from $G$ to $G^{\prime \prime}$ is uniformly continuous into the two sided uniformity on $G^{\prime \prime}$ and hence extends uniquely to a continuous map $\bar{j}$ of $\bar{G}$ onto $G^{\prime \prime}$ which is also a group homomorphism. Let $H$ be the kernel of $\bar{j}$ in $\bar{G}$, and we claim that $i\left(G^{\prime}\right)$ is dense in $H$ for if $h \in H$, there is a net $g_{\alpha} \in G$ converging to $h$. Since $j\left(g_{\alpha}\right) \rightarrow \bar{j}(h)=e$, we may find a subnet $g_{i(\beta)}$ of the $g$ 's and a net $h_{\beta}$ in $G$ with $j\left(h_{\beta}\right)=j\left(g_{i(\beta)}\right)$ and with $h_{\beta} \rightarrow e$. Then $g_{i(\beta)} h_{\beta}^{-1}$ is a net in $i\left(G^{\prime}\right)$ converging to $h$. Then as $G^{\prime}$ is assumed complete, so is $i\left(G^{\prime}\right)$, which is therefore closed in $\bar{G}$. But then $i\left(G^{\prime}\right)=H$, and it follows immediately that $G=\bar{G}$ and $G$ is complete.

Let us record now a fundamental lemma of Dixmier [9] (see also [2]) which assures the existence of good cross sections in our case. We recall that we understand by the Borel field in a metric space, the $\sigma$-field generated by the open sets.

Proposition 4. If $G$ is polonais, and $\mathrm{H}$ a closed subgroup, and $p$ the projection map to the coset space $G / H$ (which is a metric space) then there exists a Borel cross section s from $G / H$ to $G$, that is a Borel function s from $G / H$ to $G$ such that $p(s(\dot{g}))=\dot{g}$ for $\dot{g} \in G / H$.

Finally, let us also record a classic continuity theorem $[3$, p. 23] for 
homomorphisms, and a closed graph theorem [18, p. 400] which will play a major technical role.

Proposition 5 (a). Let $G_{1}$ and $G_{2}$ be separable metric groups with $G_{1}$ polonais, or more generally second category, and let $\varphi$ be a Borel homomorphism from $G_{1}$ to $G_{2}$; then $\varphi$ is continuous.

(b) Let $G_{1}$ and $G_{2}$ be polonais and let $\varphi$ be a continuous 1-1 homomorphism of $G_{1}$ onto $G_{2}$; then $\varphi$ is bicontinuous.

We now turn to a fundamental construction which we shall use throughout. We let $(X, B, \mu)$ be a $\sigma$-finite measure space with Borel field $B$ and measure $\mu$, and assume that the Borel space $(X, B)$ is countably generated (cf. [1, Chapter I]); this assures, for instance, that such function spaces as $L^{2}(X, \mu)$ are separable. Next we let $A$ be any separable metric space-later we will specialize $A$ to be a topological group. Now we define $U(X, A)$ to be the set of equivalence classes of $\mu$-measurable functions from $X$ into $A$, where two functions are equivalent if they are equal almost everywhere. Since any class contains a $B$-Borel function, we could as easily have taken equivalence classes of $B$-Borel functions and obtained the same space. We note also that $U(X, A)$ depends only on the measure class of $\mu$ and not on $\mu$ itself. Our object is to topologize $U(X, A)$ in a natural way and for this we choose a finite measure $\nu$ on $X$ equivalent to $\mu$ (which we can always do as $\mu$ is $\sigma$-finite) and choose a metric $\rho$ on $A$ in which $A$ has finite diameter. Then for $f, g \in U(X, A)$ we define

$$
\bar{\rho}(f, g)=\int_{X} \rho(f(x), g(x)) d v(x) .
$$

It should be noted that this is meaningful as $f$ and $g$ are measurable, and hence the integrand is measurable. It is also bounded and as $v$ is finite, there is no convergence problem. We have as usual, and will continue to, abuse notation and identify functions with their equivalence classes. It is trivial to verify that $\bar{\rho}$ defines a metric on $U(X, A)$, one key point being that $\bar{\rho}(f, g)=0$ if and only if $f=g$ a.e., or in other words $f=g$ in $U(X, A)$. We have the following important facts about $\bar{\rho}$.

Proposition 6. For a sequence $f_{n}$ in $U(X, A)$, the following are equivalent:

(1) $f_{n} \rightarrow f$ in the metric $\bar{\rho}$,

(2) $f_{n} \rightarrow f$ in v-measure, i.e. $\forall \epsilon, \nu\left\{x \mid \rho\left(f_{n}(x), f(x)\right)>\epsilon\right\} \rightarrow 0$ as $n \rightarrow \infty$,

(3) every subsequence of $f_{n}$ has a subsequence converging almost everywhere to $f$.

Proof. (1) $\Rightarrow(2)$ is clear since $\nu\left\{x \mid \rho\left(f_{n}(x), f(x)\right) \geqslant \epsilon\right\} \leqslant \epsilon^{-1} \bar{\rho}\left(f_{n}, f\right)$. 
Now if (2) holds, the sequence $g_{n}(x)=\rho\left(f_{n}(x), f(x)\right)$ converges to zero in $\nu$. measure and as $\nu$ is finite it follows classically that every subsequence has a subsequence converging a.e. to zero, which is (3). Finally if (3), it follows that the sequence $g_{n}(x)$ above converges to zero in measure and then by dominated convergence $\bar{\rho}\left(f_{n}, f\right) \rightarrow 0$ and so (1) is true.

We have the following important corollary of the above which follows by using statement (3).

Corollary. The topology defined on $U(X, A)$ by $\bar{\rho}$ is independent of the choice of the measure $\nu$ and depends only on the measure class of $\nu$ (or $\mu$ ) and additionally is independent of the choice of metric $\rho$ on $A$, and depends only on the topology of $A$.

It is also easy to see that if $\varphi: A \rightarrow B$ is continuous, then the induced map $\varphi^{*}: U(X, A) \rightarrow U(X, B)$ is continuous, and that if $\varphi$ is a topological embedding, that $\varphi^{*}$ is also. Moreover, if $A=\Pi A_{n}$ with the product topology, $U(X, A)=$ $\Pi U\left(X A_{n}\right)$ again with the product topology. We now have the following

Proposition 7. $U(X, A)$ is a separable metric space and is polonais if $A$ is.

Proof. Let $I=I_{n}=[0,1]$ and let $C=\Pi U_{n}$ be an infinite cube. Then as $A$ is separable, $A$ can be embedded in $C$ and hence by our remarks, $U(X, A)$ is embedded in $U(X, C)=\Pi U(X, I)$. But now $U(X, I)$ is isometric to a closed subset of the Banach space $L_{1}(X, \mu)$ which is a separable Banach space. Thus $U(X, I)$ is polonais, and in particular separable, and hence so is $U(X, C)$ and hence also $U(X, A)$. Moreover $U(X, C)$ is also evidently polonais and to show that $U(X, A)$ is polonais if $A$ is, it suffices to show that $U(X, A)$ is a $G_{\delta}$ in $U(X, C)$. Since $A$ is a $G_{\delta}$ in $C$ as it is polonais, $A=\bigcap V_{m}$ with $V_{m}$ open. It is routine to see that $U(X, A)=\bigcap U\left(X, V_{m}\right)$ and so it suffices to show that $U(X, V)$ is a $G_{\delta}$ in $U(X, C)$ if $V$ is open in $C$.

To see this, let, for any $a>0, F_{a}=\{f \mid \nu\{x \mid f(x) \in C-V\} \geqslant a\}$, and we claim that $F_{a}$ is closed. Let $f_{n} \rightarrow f$; we can, by going to subsequence, assume that $f_{n}(x) \rightarrow f(x)$ a.e., and by excluding this irrelevant null set assume that $f_{n}(x) \rightarrow f(x)$ everywhere. Then let $X_{n}=\left\{x \mid f_{n}(x) \in C-V\right\}$ and let $x \in$ $\bigcap_{n}\left(\cup_{n>m} X_{m}\right)=Y$ so that $x \in X_{m}$ for infinitely $m$, and hence $f_{m}(x) \in C-V$ for infinitely $m$, and hence $f(x) \in C-V$. It is then easy to see that $v(Y) \geqslant$ $\lim \inf v\left(X_{m}\right) \geqslant a$ and that $Y \subset\{x \mid f(x) \in C-V\}$. Thus $f \in F_{a}$ and $F_{a}$ is closed. Let $G_{a}$ be the complement of $F_{a}$ which is open and now it is immediate that $U(X, V)=\bigcap G_{1 / n}$ is a $G_{\delta}$, and we are done.

We have already observed that if $\varphi: A \rightarrow B$ is continuous, then $\varphi^{*}: U(X, A)$ $\rightarrow U(X, B)$ is continuous, but now suppose that $\varphi$ is only Borel; it is true, but 
less obvious that $\varphi^{*}$ is Borel, and we must give first a somewhat technical statement that will be useful in other contexts. Consider a space $U(X, A)$, and let $Y$ be a Borel subset of $X$, and $\phi$ a bounded Borel function from $A$ to the real numbers. Then define for $f \in U(X, A)$,

$$
G(Y, \phi)(f)=\int_{Y} \phi(f(x)) d \nu(x) .
$$

Proposition 8. The functions $G(Y, \phi)$ are Borel functions on $U(X, A)$ and define this Borel structure in the sense that it is the smallest Borel field such that all such functions are Borel.

Proof. If $\phi$ is continuous, it is easy to see using (3) of Proposition 6 that $G(Y, \phi)$ is a continuous function on $U(X, A)$. On the other hand, the class $F$ of all bounded Borel functions $\phi$ from $A$ to the real numbers such that $G(Y, \phi)$ is a Borel function is closed under bounded pointwise convergence, and as it contains all bounded continuous functions, it must contain all bounded Borel functions. Hence $G(Y, \phi)$ is a Borel function on $U(X, A)$ for each $Y$ and each $\phi$.

Finally to show that these functions define the Borel structure on $U(X, A)$, let us embed $A$ in some completion $\bar{A}$ of $A$; we note that each $\phi$ may be extended to some Borel function $\bar{\phi}$ on $\bar{A}$ to the real numbers, [18, p. 341]. Then as $U(X, A) \subset U(X, \bar{A})$ and since for $f \in U(X, A), G(Y, \phi)(f)=G(Y, \bar{\phi})(f)$, it will suffice to prove the result when $A=\bar{A}$ or when $A$ is polonais. In case $U(X, A)$ is polonais, we show that some countable subfamily does generate the Borel structure. Indeed let $Y_{i}$ range over the field generated by a countable number of generators for $B$ (the Borel field of $X$ ) and let $\phi_{j}$ run over any countable family of functions separating points of $A$. We claim then that the countable family of functions $G\left(Y_{j}, \phi_{i}\right)$ defines the Borel structure. If we note that $U(X, A)$ is polonais and hence standard as Borel space and use the fundamental separation theorem (cf. [18, p. 393]), we see that it suffices merely to show that these functions separate points of $U(X, A)$. If they did not, we would have for some $f \neq g, G\left(Y_{j}, \phi_{i}\right)(f)=G\left(Y_{j}, \phi_{i}\right)(g)$, and by the properties of the $Y_{j}$ and the definition of the functions $G$, it would follow that $G\left(Y, \phi_{i}\right)(f)=G\left(Y, \phi_{i}\right)(g)$ for all $Y$. Now if $\phi_{i}(f(x))=\phi_{i}(g(x))$ a.e. for all $i$, it follows at once that $f(x)=g(x)$ a.e. and hence $f=g$ in $U(X, A)$, so there is some $i$ so that $\phi_{i}(f(x)) \neq \phi_{i}(g(x))$ a.e. and if we put $Y^{ \pm}=\left\{x \mid \phi_{i}(f(x))-\phi_{i}(g(x)) \in R^{ \pm}\right\}$, then we cannot have $G\left(Y^{ \pm}, \phi_{i}\right)(f)=G\left(Y^{ \pm}, \phi_{i}\right)(g)$, a contradiction. This completes the proof of the lemma.

The original question we asked now becomes trivial.

COROLlaRY. If $\psi$ is a Borel function from $A$ to $B, \psi^{*}$ is a Borel function from $U(X, A)$ to $U(X, B)$. 
Proof. By the proposition, $\psi^{*}$ is Borel if and only if $G(Y, \phi) \circ \psi^{*}$ is Borel; but this function is precisely $G\left(Y, \phi \circ \psi^{*}\right)$ which is Borel by the proposition.

We come now to one of the most important facts about the spaces $U(X, A)$ and that is a version of the Fubini theorem. As we shall see, this will play a central role.

THEOREM 1. There are natural isomorphisms

$$
U(X \times Y, A) \cong U(X, U(Y, A)) \cong U(Y, U(X, A)) .
$$

Proof. The map, of course, comes by taking $F(x, y) \in U(X \times Y, A)$ and associating to it the function $x \rightarrow f_{x}$ where for each $x, f_{x}(y)=F(x, y) ; f_{x}$ is supposed to be in $U(Y, A)$. Indeed, if $F(x, y)$ is a Borel function of $x$ and $y$, then, of course, $f_{x}$ is a Borel function of $y$ and represents an element of $U(Y, A)$. We have to show that $x \rightarrow f_{x}$ is a Borel function, and to do that we use Proposition 8. All we have to do is show that $x \rightarrow G(Z, \phi)\left(f_{x}\right)$ is Borel for each $Z \subset Y$ and each bounded Borel function $\phi$ of $A$ into the reals. But $G(Z, \phi)\left(f_{x}\right)=$ $\int_{Z} \phi(f(x, y)) d \nu(y)$ is a Borel function of $x$ by the usual Fubini theorem. Finally we have to show the map $F \rightarrow\left\{f_{x}\right\}$ is well defined on equivalence classes and this is true since if $F^{1}(x, y)=F^{2}(x, y)$ a.e. in $X \times Y$, then by the usual Fubini theorem, $f_{x}^{1}=f_{x}^{2}$ a.e. $(y)$ for almost all $x$ and so the equivalence class of $F$ defines a unique element in $U(X, U(Y, A)$ ); let us call it $i(F)$. Moreover $i$ is one-one again by Fubini's theorem; and Fubini's theorem and criterion (3) of Proposition 6 show us immediately that a sequence $F_{n}$ converges to a limit $F$ if and only if $i\left(F_{n}\right)$ converges to $i(F)$ so that $i$ is bicontinuous. It remains now only to show that $i$ is onto.

To do this we remark that if $A \subset B$ and if the theorem is true for $B$, then it is true for $A$ for if we are given $f \in U(X, U(Y, A))$, we can by assumption find $F \in U(X, U(Y, B))$ with $i(F)=f$, but since $F(x, y)=f_{x}(y)$ a.e., it follows that $F$ takes values a.e. in $A$ and so is an element of $U(X, U(Y, A))$. Moreover, if the theorem is true for spaces $A_{n}$, it is by our previous remarks true for the product $\Pi A_{n}$. Therefore by the same technique that was used in Proposition 7 , it suffices to prove the theorem for $A=I=[0,1]$, the unit interval, and in this case the result is known but not quite in this language [19, Lemma 3.1]. Let us outline how that argument goes in this case. Given $f \in U(X, U(Y, I))$, fix a rectangle $M \times N \subset X \times Y$, and finite measure $\mu_{X}$ and $\mu_{Y}$ on $X$ and $Y$ equivalent to the given ones. Then define

$$
\lambda(M \times N)=\int_{X}\left\{\int_{Y} \chi_{N}\left(f_{x}(y)\right) d \mu_{Y}(y)\right\} d \mu_{X}(x)
$$

where $\chi_{N}$ is the characteristic function of $N$. Note that Proposition 8 is precisely 
what is needed to know that the integral in brackets is a Borel function of $x$, and hence that the formula has meaning. One then shows that $\lambda$ extends to a measure on $X \times Y$ and that $\lambda(K)=\int_{X}\left(\int \chi_{K(x)}\left(f_{x}(y)\right) d \mu_{Y}\right) d \mu_{X}$ where $K(x)=\{y$ : $(x, y) \in K\}$ is given by an iterated integral for any Borel $K$. Then evidently $\lambda$ is absolutely continuous with respect to $\mu_{X} \times \mu_{Y}$ and has a Radon-Nikodým derivative $F$ with respect to it, which is a Borel function on $X \times Y$. It is routine to show that $i(F)=f$ so that $F$ is the desired element of $U(X \times Y, n)$. One should consult Mackey's original paper for further details.

We might remark on several examples of our construction $U(X, A)$; if for example $X$ consists of countably many points each of positive measure, $U(X, A)$ is the infinite product $\Pi_{i=1}^{\infty} A$ of copies of $A$ with the product topology. In a sense then, $U(X, A)$, in general, may be thought of as a direct integral of copies of $A$. One could imagine being given a function $x \rightarrow A_{x}$ from $X$ into sets $A_{x}$ and attempting to form an analogous direct integral of the $A_{x}$ 's as one does with Hilbert spaces. No doubt this could be done under suitable hypotheses, but as no need has been shown for such a construction, we will not proceed.

Up until now the space $A$ has just been a set with a separable metric topology. Let us now specialize to the case of interest when $A$ is a polonais group, especially an abelian one. It follows immediately from Proposition 6 that the pointwise multiplication of functions makes $U(X, A)$ into a topological group and by Proposition 7, a polonais group. As an example, we note that if $A=T$ is the circle group, then $U(X, T)$ may be identified as the group of all unitary operators in the von Neumann algebra of multiplication operators on $L_{2}(X)$, and one immediately sees that the topology we have constructed is precisely the strong operator topology; incidentally this is in effect why this construction is so useful. Also, if $X=R$, then $U(X, R)$ is a (nonlocally convex) topological vector space which has been introduced in integration theory (cf. [11]); one might point out that this space has no continuous linear functionals. A related point which we leave as an exercise is the observation that if $X$ is nonatomic then $U(X, T)$ has no nonconstant homomorphisms to $T$, or in other words the "Pontrjagin dual group" is reduced to a single point. Another interesting example of this construction is to choose $A=Z_{2}$, the group (field) of two elements. Then $U\left(X, Z_{2}\right)$ can easily be seen to be a topological ring which is nothing other than the measure algebra of $(X, \mu)$ equipped with its usual topology.

Let us note some additional properties of the groups $U(X, A)$; one of the most useful is the following:

Proposition 9. If $1 \rightarrow A^{\prime} \rightarrow A \rightarrow A^{\prime \prime} \rightarrow 1$ is an exact sequence of polonais groups, commutative or not, then $1 \rightarrow U\left(X, A^{\prime}\right) \rightarrow U(X, A) \rightarrow$ $U\left(X, A^{\prime \prime}\right) \rightarrow 1$ is an exact sequence of polonais groups. 
Proof. The continuity of maps and exactness except at the final point are routine or follow from previous comments. If $\varphi$ is the map from $A$ to $A^{\prime \prime}$, then the only point at issue is the surjectivity of the induced map $\varphi_{*}$, but once one observes that there is a Borel cross section $s$ from $A^{\prime \prime}$ into $A$, we have an immediate way to find a preimage of an $f \in U\left(X, A^{\prime \prime}\right)$, namely $s \circ f$.

In this development we have taken as the basic class of groups, the polonais groups which from some points of view may be too large a class. In fact a rather more natural class of groups from our point of view, although not from others would be the subclass of $P^{\prime}$ consisting of those topological groups which may be realized as (closed) groups of unitary operators on a separable Hilbert space. This class is closed under taking subgroups, products, the construction $U\left(X,{ }^{\cdot}\right)$ and includes all locally compact separable groups, but we do not know if it is closed under quotients, and this is a fatal drawback if not true.

There is one complication in this theory which as we shall see, simply cannot be avoided and this comes about as follows: We have polonais groups $A$ and $B$ (say abelian) together with a continuous homomorphism $j$ from $A$ into $B$. The quotient group $B / j(A)$ will be of interest and significance even though in many cases $j(A)$ is not closed in $B$. This quotient group would be polonais if $j(A)$ were closed but in general it is some non-Hausdorff topological group, arising in some sense as the "quotient" of two polonais groups. It will be somewhat useful to talk about such objects which with some trepidation one might call pseudopolonais groups. We would define such objects as triples $C=(A, B, j)$ where $A$ and $B$ are polonais and $j$ is a continuous-homomorphism of $A$ into $B$, subject to an appropriate equivalence relation which we shall not pursue at this moment. One would think of this equivalence class as representing the group $B / j(A)$ and an ordinary polonais group $B$ could be represented by the class of $(0, B, 0)$. For our purposes the most important thing to define is what is meant by a Borel map of a space $X$ into such an object; for this we consider the groups $B(X, A)$ and $B(X, B)$ the Borel maps of $X$ into $A$ and $B$ respectively. There is an induced map $j^{*}$ from the first to the second and we define

$$
B(X, C)=\operatorname{coker}\left(j^{*}\right)=B(X, B) / j^{*}(B(X, A)) .
$$

We proceed in a similar way to define $U(X, C)$, the equivalence classes of measurable maps of $X$ in $C$ in an analogous way as $U(X, B) / j^{*}(U(X, A))$ so that $U(X, C)$ is a pseudo-polonais group represented by the triple $\left(U(X, A), U(X, B), j^{*}\right)$. One remark in this regard will be useful later on.

Proposition 10. The closure of the identity element in $U(X, C)$ consists of the image in $U(X, B) / j^{*}(U(X, A))$ of all functions taking values a.e. in the closure of the range of $j, \overline{j(A)}$. 
Proof. The subgroup $W$ of $U(X, B)$ described is clearly a closed subgroup of $U(X, B)$ containing $j^{*}(U(X, A))$ and all we have to do is to show that it is precisely the closure of $j^{*}(U(X, A))$. Suppose $f \in W$; then evidently $f$ can be approximated as closely as we please by elements of $W$ which take only a finite number of values in $\overline{j(A)}$, and it suffices to show that each such function is in the closure of $j^{*}(U(X, A))$, but this is clear.

This completes our discussion of the kinds of groups to be considered. It was somewhat extended but this material is apparently not to be found anywhere else. We now turn to some similar results on group actions.

3. Suppose that $G$ is locally compact, and suppose that $A$ is a polonais group, where for simplicity we denote the class of abelian such $A$ by $P$. We say that $G$ operates on $A$ or that $A$ is a $G$ module if we have a homomorphism $\rho$ of $G$ into the automorphisms of $A$, written $\rho(g)(a)=g \cdot a$ so that $(g, a) \rightarrow g \cdot a$ is jointly continuous. The group $G$ could be any topological group but we have to restrict to locally compact groups in this paper in order to obtain nontrivial results. It would, of course, be more satisfying to have $G$ polonais, but even in this case we do not see how to proceed to develop a reasonable cohomology theory. We denote the class of abelian polonais $G$-modules by $P(G)$ and note that the admissible maps from $A$ to $B$ are the continuous homomorphisms from $A$ to $B$ intertwining the action of $G$. One defines submodules, quotient modules, products, finite or infinite in the obvious way, and in addition if $A \in P(G)$, then $U(X, A) \in P(G)$ with the same for nonabelian $A$ where we define $(g \cdot \phi)(x)=$ $g \cdot(\phi(x))$ for $\phi \in U(X, A)$; in fact we could consider a family of actions of $G$ on $A$ indexed by points in $x$, varying sufficiently smoothly in $x$ and make a similar definition. It is convenient on many occasions to be able to start with an action of the group $G$ on the group $A$ which satisfies the algebraic conditions necessary for $\boldsymbol{A}$ to be a $\boldsymbol{G}$-module together with very weak continuity conditions, and then deduce the full continuity conditions. The following is an example of one such result:

Proposition 11. Let $G$ and $A$ be polonais with $G$ acting algebraically as a group of automorphisms of $A$ such that $(g, a) \rightarrow g \cdot a$ is a Borel function in each of its variables separately. Then it is jointly continuous, so that $A$ is a $G$ module.

Proof. The kind of reasoning here is completely standard. We note that $a \rightarrow g \cdot a$ is a Borel automorphism of $A$ and since $A$ is polonais, it is continuous. We now show that $g \rightarrow g \cdot a$ is continuous, and for this, select a residual set $P$ in $G$ so that $g \rightarrow g \cdot a$ restricted to $P$ is continuous. Then let $g_{n} \rightarrow g_{0}$ in $G$ and consider the residual sets $P g_{n}^{-1}$ and let $P_{0}=\bigcap_{n=0}^{\infty} P g_{n}^{-1}$ which is also resi- 
dual. Let $g \in P_{0}$ so that $g g_{n} \in P$ for $n=0,1,2, \ldots$ Since $g g_{n} \rightarrow g g_{0}$, we know $\left(g g_{n}\right) \cdot a \rightarrow\left(g g_{0}\right) \cdot a$ and by the continuity of the automorphism $b \rightarrow$ $g \cdot b$, we see that $g_{n} \cdot a \rightarrow g_{0} \cdot a$ as desired.

We have now shown that $g \cdot a$ is separately continuous, whence by $[18, p$. 285] it is a joint Borel function of both its variables. It follows then as in [29] that it is jointly continuous.

One of the most significant types of $G$ modules arises from point actions of the group $G$. Specifically suppose that $G$ acts on an analytic Borel space $X$ as a Borel transformation group [1] and suppose that it leaves some finite measure $\mu$ quasi-invariant. Then for any polonais $A$ we define an action of $G$ on $U(X, A)$ by $(s \cdot f)(x)=f\left(s^{-1}(x)\right)$ where $s(x)$ is the action of $s$ on a point $x \in X$ and where we understand $(X, \mu)$ to be the measure space in question. It follows from the fact that $\mu$ is quasi-invariant that $s \cdot f$ is well defined.

Proposition 12. With above definition $U(X, A)$ becomes a G-module.

Proof. We show that $f \rightarrow s \cdot f$ is Borel and in fact continuous; in fact let $f_{n} \rightarrow f$ so that by Proposition 6 , a given subsequence has some subsequence converging almost everywhere to $f$, say for $x \notin N$. Then since $s \cdot N$ is a null set also, the sequence $s \cdot f_{n}$ has the property that a given subsequence has a subsequence, namely the one above which converges to $s \cdot f$ for $x \notin s \cdot N$, and hence $s \cdot f_{n} \rightarrow f_{n}$ again by Proposition 6.

On the other hand, let us fix some $f \in U(X, A)$ and consider one of the functions $G(Y, \phi)$ of Proposition 8 which generate the Borel field on $U(X, A)$. To show $s \rightarrow s \cdot f$ is Borel it is enough to show that

$$
s \rightarrow G(Y, \phi)(s \cdot f)=\int_{X} \chi_{Y}(x) \phi\left(f\left(s^{-1}(x)\right) d \mu(x)\right.
$$

is a Borel function. However, we simply note that the integrand is a joint Borel function of $s$ and $x$ and therefore the integral with respect to $x$ is a Borel function of $s$ by the standard version of Fubini's theorem. Our result now follows from the previous proposition.

For example, if we take $A=Z_{2}$, then we see that the measure algebra of $(X, \mu)$ is a $G$-module, complementing the discussion in [25]. Of course, the right continuity assumption for the point realization theorem in [25] is that a measure algebra $M$ is a topological $G$-module so that $G$ acts by algebra automorphisms.

In the above discussion we have taken $A$ to be a group, but the result immediately extends to separable metric spaces since any such $A$ can be embedded topologically in an infinite torus $S$ and so $U(X, A) \subset U(X, S)$ and if $G$ acts as a topological transformation group on $U(X, S)$, it does also on $U(X, A)$.

Perhaps one of the most important examples of the construction above is 
the case when $X=G$ itself with a finite measure equivalent to Haar measure. Then if $A$ is polonais, we denote the module $U(X, A)$ by $I(A)$. This $G$-module will play a key role for us; in fact it would be fair to call this module the regular representation of $G$ with coefficients in $A$. It is also a special case of the more general notion of induced modules which we shall be defining presently, however, first let us investigate $I(A)$. Suppose that in addition that $A$ has already the structure of a $G$-module. Then for $a \in A$, consider $F_{a}(s)=s^{-1} \cdot a$ which as one sees is an element of $I(A)$.

Proposition 13. The map $a \rightarrow F_{a}$ is a G-module isoinorphism of $A$ onto a closed submodule of $I(A)$.

Proof. That $F_{g \cdot a}=g \cdot F_{a}$ is routine; moreover if $a(n) \rightarrow a$, it is clear that $F_{a(n)} \rightarrow F_{a}$ everywhere so that the map is continuous. On the other hand if $F_{a(n)} \rightarrow F_{a}$ in $U(G, A)=I(A)$, then for any subsequence of the $a(n)$, some subsequence $b(m)$ would have the property that $F_{b(m)}(s) \rightarrow F_{a}(s)$ for almost all $s$, and hence trivially $b(m) \rightarrow a$. It follows by elementary theorems then that $a(n)$ converges to $a$. Thus $a \rightarrow F_{a}$ is a homeomorphism as desired and its range is automatically closed as it is complete, and the proof is finished.

We now define $i(a)=F_{a}$ and let $U(A)$ be the cokernel of $i$ so that $U(A)$ is a $G$-module and we have an exact sequence $0 \rightarrow A \rightarrow I(A) \rightarrow U(A) \rightarrow 0$.

Proposition 14. I and $U$ are covariant functors on $P(G)$ to $P(G)$ and carry short exact sequences into short exact sequences.

Proof. This routine calculation is omitted.

On the other hand, the following calculation is somewhat more involved and will be most useful.

Proposition 15. For A polonais, we have a canonical isomorphism of G-modules $U(I(A)) \simeq I(U(A))$.

Proof. We note from their definitions both of the modules in the statement of the proposition are quotients of $I(I(A))$ by subgroups which are both isomorphic to $I(A)$ but the copies are embedded differently. Now $I(I(A))=$ $U(G, I(A))=U(G, U(G, A))$ which by our Fubini theorem may be identified with $U(G \times G, A)$. Let us agree that the first variable refers to the $G$ in $U(G, I(A))$ so that the action of $G$ on $U(G \times G, A)$ under this identification is $(s \cdot F)(x, y)=F\left(s^{-1} x, y\right)$. Now $U(I(A))$ is obtained from $I(I(G))$ by factoring by elements of the form $s \rightarrow s^{-1} \cdot f$ for $f \in I(A)$ or in terms of functions of two variables, all functions of the form $F(s, t)=\left(s^{-1} \cdot f\right)(t)=f(s t)$ for some $f \in I(A)$; call this subgroup $B_{1}$. On the other hand $I(U(A))$ is obtained by factoring by the subgroup of all elements of the form $s \rightarrow f(s)$ where $f(s)$ is the image 
of $A$ sitting in $I(A)$ and hence has the form $t \rightarrow t^{-1} \cdot a$, and so the group can be described as the set of all functions of the form $F(s, t)=t^{-1} \cdot a(s)$ where $a$ is some function in $I(A)$ and where $t \cdot a$ is the given $G$-module structure on $A$. Call this subgroup $B_{2}$, and note that it depends on the $G$-module structure of $A$ whereas $B_{1}$ did not. Our problem then is to find a $G$-module automorphism $K$ of $U(G \times G, A)$ with the action as described above which carries $B_{1}$ onto $B_{2}$. It may be verified that $(K F)(s, t)=t^{-1} \cdot F\left(s t^{-1}, t\right)$ is such a mapping, and this completes the proof.

We shall now consider an extension of the construction $I(A)$ which will provide us with the analogue of induced modules in the case of finite groups. We suppose that $H$ is a closed subgroup of $G$ and that $A$ is a polonais $H$-module; we shall construct the induced module $I_{H}^{G}(A)$, a polonais $G$-module. We first consider $I(A)$ and then let $I_{H}^{G}(A)$ be the subset of $I(A)$ consisting of all functions $f$ such that $f(s h)=h^{-1} \cdot f(s)$ for almost all pairs $(s, h)$ in $G \times H$, the product of Haar measure being understood. One has to comment that this makes sense since $I(A)$ consists of equivalence classes of functions instead of functions; or more precisely, let $f_{1}$ satisfy the condition and let $f_{2}=f_{1}$ a.e.; then we must show that $f_{2}$ satisfies the condition. This is clear, for if $N=\left\{s, f_{1}(s) \neq f_{2}(s)\right\}$ and $M=\left\{(s, h): f_{h}(s h) \neq h^{-1} \cdot f_{2}(s)\right\}$, and if $\phi$ is the map $(s, h) \rightarrow s \cdot h$, then $\phi^{-1}(N)$ is also a null set and it is clear that $\left\{(s, h): f_{2}(s h) \neq h^{-1} \cdot f_{2}(s)\right\} \subset$ $M \subset \phi^{-1}(N)$, and so $f_{2}$ satisfies the condition.

Proposition 16. $I_{H}^{G}(A)$ is a closed G-invariant subgroup of $I(A)$ and hence is a polonais G-module.

Proof. The $G$-invariance is clear and if $f_{n} \in I_{H}^{G}(A)$ and $f_{n} \rightarrow f$, there is some subsequence converging pointwise a.e. so we may assume $f_{n}(s) \rightarrow f(s)$, $s \notin N$. Then if the defining condition on $f_{n}$ fails on the null set $M_{n} \subset G \times H$, we see that $\left\{(s, h) \mid f(s h) \neq h^{-1} \cdot f(s)\right\} \subset \bigcup_{n} M_{n} \cup \phi^{-1}(N)$, which as above is a null set.

As in the case of unitary representations, it is sometimes helpful to be able to identify the underlying group structure of $I_{H}^{G}(A)$ with something more common. Indeed, let $c$ be a Borel cross section from $G / H$ into $G$, and for any $f \in$ $U(G / H, A)$, we define $\bar{f}$ by $\bar{f}(c(s) h)=h^{-1} f(s)$ for $\dot{s} \in G / H$ and $h \in H$, where we implicitly place on $G / H$, any quasi-invariant measure.

Proposition 17. The function $\bar{f}$ on $G$ is in $I_{H}^{G}(A)$ and the map $f \rightarrow \bar{f}$ defines an isomorphism of topological groups from $U(G / H, A)$ onto $I_{H}^{G}(A)$.

Proof. If $f$ is a Borel function, it is clear that $\bar{f}$ is a Borel function on $G$ since the map $(G / H) \times H$ into $G$ given by $(\dot{s}, h) \rightarrow c(s) h$ is known to be a Borel isomorphism. Moreover, if $f$ is changed on a null set, $\bar{f}$ only changes on a null 
set so the map $f \rightarrow \bar{f}$ is well defined. It is clear that $\bar{f} \in I_{H}^{G}(A)$ since the particular function $\bar{f}$ satisfies the defining condition everywhere. Again a straightforward use of criterion (3) of Proposition 6 for convergence of sequences implies that $f \rightarrow \bar{f}$ is a continuous function, and since it is clearly injective, it will suffice to show it is surjective and then appeal to the standard closed graph theorem to conclude that it is an isomorphism.

Consider therefore some $F \in I_{H}^{G}(A)=B$. Since $F(s h)=h^{-1} \cdot F(s)$ holds for almost all pairs, it follows by Fubini that there is a null set $N$ in $G$ so that if $s \notin N, F(s h)=h^{-1} \cdot F(s)$ holds for almost all $h \in H$. We define a function $F^{\prime}$ on $N^{c} \times H$ by $F^{\prime}(s, h)=h^{-1} \cdot F(s)$ for $s \notin N, h \in H$. Suppose now that $s_{1} \notin N$ and $s_{1}=s \cdot h_{1}$ for $s \notin N$. Then

$$
h^{-1} \cdot F\left(s_{1}\right)=F\left(s_{1} h\right)(\text { a.e. } h)=F\left(s h_{1} h\right)=h^{-1} h_{1}^{-1} \cdot F(s)
$$

for almost all $h_{1} h$ and hence for almost all $h$. Thus $h^{-1} \cdot F\left(s_{1}\right)=h^{-1} h_{1}^{-1} F(s)$ for almost all $h$ and since both sides are continuous in $h$, it holds for all $h$, so we see that $F^{\prime}\left(s h_{1}, h\right)=F^{\prime}\left(s, h_{1} h\right)$ when $s$ and $s h_{1}$ are in $N^{c}$. We then define a function $F^{\prime \prime}$ on $L=\{y: \exists h \in H, y h \notin N\} \supset N^{c}$ by $F^{\prime \prime}(y)=F^{\prime \prime}(s h)=$ $F^{\prime}(s, h)$ if $s h=y \in L$ and $s \notin N$, which is well defined by the above. Clearly $F^{\prime \prime}$ is a Borel function and $F^{\prime \prime}(s h)=h^{-1} \cdot F^{\prime \prime}(s)$. We extend the definition of $F^{\prime \prime}$ to the complement of $L$ in any Borel way so that $F^{\prime \prime}(s h)=h^{-1} \cdot F^{\prime \prime}(s)$ for all $h$ and all $s$, and $F^{\prime \prime}=F$ a.e. Then clearly $F^{\prime \prime}$ is of the form $\bar{f}$ for some measurable function $f$ on $G / H$. This shows that the map is onto and we are done.

We shall note some properties of our induced modules, the first of which is the validity of inducing in stages; let $G \supset K \supset H$.

Proposition 18. There is a canonical isomorphism of $G$ modules between $I_{H}^{G}(A)$ and $I_{K}^{G}\left(I_{H}^{K}(A)\right)$.

Proof. We note that as measure spaces $G / H \simeq G / K \times K / H$ by appropriate choice of cross sections. Then by the previous result, we may identify $I_{H}^{G}(A)$ as a polonais group with $U(G / H, A)$, and the second with $U(G / K, U(K / H, A))$ which in turn by our Fubini theorem (Theorem 1) is isomorphic to $U(G / K \times K / H, A)$ which in turn is identified with $U(G / H, A)$. This simple observation implies that the natural map, motivated by pure algebra, from $I_{H}^{G}(A)$ to $I_{K}^{G}\left(I_{H}^{K}(A)\right)$ is well defined and is in fact an isomorphism of topological groups. The fact that this map intertwines the $G$-actions is routine, and so modulo some routine details which we omit, the result is established.

Proposition 19. The map $A \rightarrow I_{H}^{G}(A)$ is a covariant functor from $H$ modules to G-modules which sends exact sequences to exact sequences. Moreover the $G$ set of fixed elements of $I_{H}^{G}(A),\left(I_{H}^{G}(A)\right)^{G}$ is canonically isomorphic to the set of $H$-fixed elements of $A, A^{H}$. 
Proof. The first assertion is clear and follows the lines of Proposition 9. For the second assertion, suppose that $a \in A^{H}$; and then define $\bar{a} \in I_{H}^{G}(A)$ by $\bar{a}(g)=a$. Since $h \cdot a=a$, we see that $\bar{a} \in I_{H}^{G}(A)$ and is clearly a $G$-invariant element as it is a constant function. This map $a \rightarrow \bar{a}$ is clearly a continuous injection of $A^{H}$ into $\left(I_{H}^{G}(A)\right)^{G}$ and we need only show it is onto. Let $f$ be in the latter group so that $f(g s)=f(s)$ for almost all $s$ for each $g$. By Fubini's theorem it follows that there is a null net $N$ in $G$ so that if $s \notin N, f(g s)=f(s)$ for almost all $g$, so that $f$ is in fact equal almost everywhere to a constant, $f(s)=a$. Then since $f \in I_{H}^{G}(A), h \cdot a=a$ for almost all $h$ and by continuity, for all $h$. Thus $a \in A^{H}$ and $f=\bar{a}$ as defined above and we are done.

4. At this point we have discussed the category of groups and modules to be considered together with a variety of constructions on them and we now at last turn to the construction of cohomology groups. As we have remarked, the program is to obtain analogues of the Eilenberg-Mac Lane groups in a way extending the groups defined in [29] and [30]. We assume some familiarity with these groups already and their properties and therefore in this context we will begin by writing down some axioms that we would want such cohomology groups to satisfy.

Definition. Let $G$ be locally compact separable; then a cohomological functor on $P(G)$, the category of polonais commutative $G$-modules is a sequence $H^{n}(G, A), n=0,1,2, \ldots$, of covariant functors on $P(G)$ to the category of abelian groups such that

(1) for each short exact sequence $0 \rightarrow A^{\prime} \rightarrow A \rightarrow A^{\prime \prime} \rightarrow 0$ in $P(G)$, there is the usual long exact sequence of cohomology

$$
\begin{aligned}
0 \rightarrow H^{0}\left(G, A^{\prime}\right) & \rightarrow H^{0}(G, A) \rightarrow H^{0}\left(G, A^{\prime \prime}\right) \stackrel{\partial_{0}}{\longrightarrow} H^{\prime}\left(G, A^{\prime}\right) \\
& \rightarrow \cdots \rightarrow H^{r}(G, A) \rightarrow H^{r}\left(G, A^{\prime \prime}\right) \stackrel{\partial_{r}}{\longrightarrow} H^{r+1}\left(G, A^{\prime}\right) \rightarrow \cdots
\end{aligned}
$$

with coboundary operators which are functoral.

(2) $H^{0}(G, A)=A^{G}$, the $G$-fixed points of $A$.

(3) $H^{r}(G, I(A))=(0)$ for all $r \geqslant 1$, and all $A$.

Some comments on this definition are clearly in order. The algebraic conditions in the initial specification and in (1) are entirely routine and would be expected of any cohomology groups worth the name. Assumption (2) is also completely routine and comes directly from all previous experience with group cohomology. On the other hand, the vanishing axiom (3) is a nontrivial restriction which really gives the theory its flavor and its strength. It can be motivated from the case of finite groups where it is a theorem based on other axioms plus the fact that in that case there is no question about what the cohomology groups should be. Moreover, the condition is motivated by one's very plausible feeling 
that the regular representation ought to have trivial cohomology, at least in dimension one and then mutatis mutandi in all higher dimensions also. No doubt one could choose a different vanishing axiom which might lead to different cohomology groups, and so the answer to the question of why we choose this one is that cohomology groups which result turn out to be of interest and to solve a number of questions which arise in the theory of group representations and the theory of operator algebras. In other words the proof is in the applications of our construction. Of course in this discussion we have been talking of the cohomology groups arising from these axioms. We now make this unicity theorem precise; the proof is entirely standard. Momentarily we come to the existence of groups satisfying these axioms which is a somewhat more difficult question.

THEOREM 2. Let $H_{1}^{*}(G, \cdot)$ and $H_{2}^{*}(G, \cdot)$ be cohomological functors on $P(G)$ satisfying the axioms above. Then there exists a unique isomorphism of functors $H_{1}^{*} \rightarrow H_{2}^{*}$ compatible with the given isomorphism in dimension 0 , so in particular $H_{1}^{n}(G, A) \simeq H_{2}^{n}(G, A)$ for all $n$ and all $A$.

Proof. We are given the isomorphism in dimension zero, and suppose by induction that we have constructed an isomorphism in dimensions $i \leqslant n-1$, and we show how to extend it to $i=n$. For $A \in P(G)$, consider the short exact sequence $0 \rightarrow A \rightarrow I(A) \rightarrow U(A) \rightarrow 0$ of $\S 3$. Then if $n \geqslant 2$, we know by our axioms that $H_{i}^{n}(G, A)=H_{i}^{n-1}(G, A)=(0)$ for $i=1,2$ and then the long exact sequences read as follows:

$$
\begin{gathered}
0 \rightarrow H_{1}^{n-1}(G, U(A)) \rightarrow H_{1}^{n}(G, A) \rightarrow 0 \\
\downarrow \\
0 \rightarrow H_{2}^{n-1}(G, U(A)) \rightarrow H_{2}^{n}(G, A) \rightarrow 0,
\end{gathered}
$$

where the left-hand vertical arrow comes from the inductive hypothesis. It is clear that one has an isomorphism on the right, $H_{1}^{n}(G, A) \rightarrow H_{2}^{n}(G, A)$ as desired. Moreover it is unique subject to functorality and subject to uniqueness in dimension $n-1$. Thus the uniqueness is established by induction at the same time as existence. It remains to consider the case $n=1$ and here the long exact sequences read

$$
0 \rightarrow A^{G} \rightarrow I(A)^{G} \stackrel{f}{\rightarrow} U(G)^{A} \rightarrow H_{i}^{1}(G, A) \rightarrow 0,
$$

and it is clear that $H_{i}^{1}(G, A)$ is unique and has to be the cokernel of the map $f: I(A)^{G} \rightarrow U(A)^{G}$. This completes the proof.

It is clear from the above that everything is determined by $H^{0}$, but it is perhaps better to also describe it in terms of $H^{1}$ and the functor $U$. Indeed let $U_{n}(A)=U(U(\cdots(A) \cdots)) n$-times-grantedly a very complicated object.

COROLlARY. We have isomorphisms $H^{n}(G, A) \simeq H^{1}\left(G, U_{n-1}(A)\right)$ for 
any cohomological functor for every $n$ and every $A$.

The point of this comment is that any sensible group cohomology functor in dimension one virtually has to be on heuristic grounds the group of continuous homomorphisms of $G$ into $A$ if $G$ acts trivially, or the equivalence classes of continuous crossed homomorphisms if the action is nontrivial. The above then gives a description of cohomology groups in all dimensions in terms of crossed homomorphisms.

We turn to the question of existence; although presumably one could in fact use the previous corollary as a definition and prove the axioms, it is essential for our purposes to proceed directly via cochain complexes. In fact we shall construct two cochain complexes. For $A \in P(G)$, let $C^{n}(G, A)$ denote the set of all Borel functions from $G^{n}$ into $A$. We equip this with the usual coboundary operator [16], [29],

$$
\begin{aligned}
\left(\delta^{n} f\right)\left(s_{1}, s_{2}, \ldots, s_{n+1}\right) & =s_{1} \cdot f\left(s_{2}, \ldots, s_{n+1}\right)-f\left(s_{1} s_{2}, s_{3}, \ldots, s_{n+1}\right) \\
& +f\left(s_{1}, s_{2} s_{3}, \ldots, s_{n+1}\right)+\cdots \\
& +(-1)^{n} f\left(s_{1}, \ldots, s_{n} s_{n+1}\right) \\
& +(-1)^{n+1} f\left(s_{1}, \ldots, s_{n}\right)
\end{aligned}
$$

where we have written $A$ additively. Secondly let us define $C^{n}(G, A)$ to be $U\left(G^{n}, A\right)$ in the sense of $\S 2 ; \underline{C}^{n}(G, A)$ can of course be obtained from $C^{n}(G, A)$ by identifying $n$-cochains which agree almost everywhere. We now define the coboundary operator $\underline{\delta}^{n}$ by exactly the same formula. We should point out that $\underline{C}^{n}(G, A)$ are polonais groups (whereas there is simply no way at all to give $C^{n}(G, A)$ a reasonable such structure if $G$ is not discrete). Finally we note that the possibility that the complex $\underline{C}^{n}(G, A)$ would work at all is suggested to us by the final theorem in [23].

Proposition 20. $C^{*}(G, A)$ with $\delta^{*}$ and $\underline{C}^{*}(G, A)$ with $\underline{\delta}^{*}$ are complexes. Moreover each $\underline{\delta}^{n}$ is a continuous homomorphism.

Proof. The assertions for $\delta^{n}$ are all entirely straightforward and in effect are in [29]. We add a few comments about $\underline{\delta}^{n}$, the first of which is that it is well defined; more specifically if $f=g$ a.e., then one sees that $\delta^{n}(f)=\delta^{n}(g)$ a.e. the key observation being that if $\varphi(x, y)=x y$ then the inverse image under $\varphi$ of a null set in $G$ is a null set in $G \times G$. Thus we can define $\delta^{n}(f), f \in$ $\underline{C}^{n}(G, A)$, as the equivalence class of $\delta^{n}(f)$ for any $f \in \underline{f}$. Finally that $\underline{\delta}^{n}$ is continuous follows at once from our remark above on null sets and criterion (3) of Proposition 6 for convergence of sequences in spaces $U(X, B)$.

We denote by $Z^{n}(G, A)\left(\underline{Z}^{n}(G, A)\right)$ the kernel of $\delta^{n}\left(\underline{\delta}^{n}\right)$, the $n$-cocyles, 
and by $B^{n}(G, A)\left(\underline{B}^{n}(G, A)\right)$ the image of $\delta^{n-1}\left(\delta^{n-1}\right)$, the $n$-coboundaries, and by $H^{n}(G, A)\left(\underline{H}^{n}(G, A)\right)$ the quotient group which we call the $n$th cohomology group of $G$ with coefficients in $A$.

Proposition 21. The functors $A \rightarrow H^{n}(G, A)$ and $A \rightarrow \underline{H}^{n}(G, A)$ satisfy conditions (1) and (2) in the definition of cohomological functor. Moreover there is a natural mapping of functors $H^{n}(G, A) \rightarrow \underline{H}^{n}(G, A)$.

Proof. If $A, B \in P(G)$ and $\phi \in \operatorname{Hom}(A, B)$, there is an induced map $C^{n}(G, A) \rightarrow C^{n}(G, B)$ by $f \rightarrow \phi \circ f$ and similarly for $\underline{C}^{n}$, which commutes with the coboundary maps. Thus there are induced maps on cohomology which clearly make these covariant functors. Now if $0 \rightarrow A^{\prime} \rightarrow A \rightarrow A^{\prime \prime} \rightarrow 0$ is a short exact sequence in $P(G)$, we have observed in [29] and also in Proposition 9 that

$$
0 \rightarrow C^{n}\left(G, A^{\prime}\right) \rightarrow C^{n}(G, A) \rightarrow C^{n}\left(G, A^{\prime \prime}\right) \rightarrow 0
$$

and also $\underline{C}^{n}(G, \cdot)$ are exact. It follows immediately by standard theorems that we have long exact sequences of cohomology as in the definition.

Finally we show $H^{0}(G, A) \simeq \underline{H}^{0}(G, A) \simeq A^{G}$. Since $B^{0}=(0)$ we show this for $Z^{0}$ and $\underline{Z}^{0}$. Now $A=C^{0}(\bar{G}, A)=\underline{C}^{0}(G, A)$ and $(\delta \cdot a)(s)=s \cdot a-a$, so that $a \in Z^{0}(\bar{G}, A) \Longleftrightarrow s \cdot a=a$ for all $s$ or in other words $a \in A^{G}$. On the other hand, $a \in \underline{Z}^{0}(G, A) \Longleftrightarrow s \cdot a=a$ for almost all $s$, but by continuity this is the same as for all $s$ and so $a \in A^{G}$ and this shows (2). Finally as we have already remarked, the map $C^{n}(G, A) \rightarrow \underline{C}^{n}(G, A)$ which associates to each $f$ its equivalence class provides natural homomorphisms commuting with the coboundary maps and hence functoral maps $H^{n}(G, A) \rightarrow \underline{H}^{n}(G, A)$ which as we have seen are isomorphisms in dimension zero.

Before we proceed further it will be useful to investigate these groups in dimension one; as we have remarked, any respectable cohomological functor should have $H^{1}(G, A)$ equal to continuous crossed homomorphisms modulo principal ones. On the other hand $\underline{Z}^{1}(G, A)$, say, consists of measurable functions $f$ from $G$ to $A$ such that $f\left(s_{1} s_{2}\right)=s_{1} \cdot f\left(s_{2}\right)+f\left(s_{1}\right)$ for almost all pairs $\left(s_{1}, s_{2}\right)$. The following closes the gap.

THEOREM 3. Let $G$ be locally compact separable, and let $H$ be a separable metric group with $G$ acting as automorphisms on $H$. Let $\varphi$ be any measurable map $G \rightarrow H$ such that $\varphi(x y)=x \cdot \varphi(y)(\varphi(x))$ for almost all pairs $x$ and $y$. Then there exists a unique crossed homomorphism $\varphi_{0}$ from $G$ to $H$ agreeing with $\varphi$ almost everywhere. Moreover $\varphi_{0}$ is continuous.

Proof. For convenience let us replace $H$ by the semidirect product $H \cdot G$ defined using the action of $G$ on $H$, and let us replace $\varphi$ by $\varphi^{\prime}(g)=(\varphi(g), g)$. Then $\varphi^{\prime}$ is an ordinary homomorphism and if we prove the theorem for $\varphi^{\prime}$ it will 
follow for $\varphi$. In other words, we assume hereafter that there is no action of $G$ on $H$ and that $\varphi$ is an ordinary homomorphism. For simplicity we may also assume that $\varphi$ is Borel.

We now define $U=\{y: \varphi(x) \varphi(y)=\varphi(x y)$ for almost all $x\}$. By a straightforward application of Fubini's theorem, $U$ is a Borel set in $G$ and is conull. Suppose that $x, y$, and $x y$ are all in $U$; then for almost all $z$,

$$
\begin{aligned}
\varphi(z) \varphi(x) \varphi(y) & =\varphi(z x) \varphi(y) \quad(\text { as } x \in U) \\
& =\varphi(z x y) \quad \text { (for almost all } z x \text { as } y \in U) \\
& =\varphi(z) \varphi(x y) \quad \text { for almost all } z \text { as } x y \in U .
\end{aligned}
$$

In particular we can find a $z$ for which all three equalities hold and we discover that $\varphi(x) \varphi(y)=\varphi(x y)$. For simplicity we may also assume that $\varphi(e)=e$ so that $e \in U$.

Now if $a_{i}$ is any finite (or countable) sequence of elements in $G, U^{-1} U$ $\bigcap_{i=1}^{\infty} a_{i} U$ is conull and hence in particular nonvoid, so that $U$ satisfies the hypotheses of Lemma 6 of [33]. The conclusion of this lemma is as follows: We form the abstract group $G$ which is specified by generators and relations. The generators are denoted $(x)$, one for each $x \in U$ and are subject to all relations of the form $(x)(y)=(x y)$ whenever $x, y$, and $x y$ are all in $U$. It is clear that $(x) \rightarrow x$ extends to a homorphism of $\dot{G}$ into $G$ and the lemma says that this is in fact an isomorphism. It is also obvious in this context that $G=U \cdot U$, and we define a map $\psi$ from $U \times U$ to $H$ by $\psi(x, y)=\varphi(x) \varphi(y)$. Now let $p$ be the mapping of $U \times U$ onto $G$ defined by $p(x, y)=x y$. Now Weil's result together with the fact that $\varphi(x) \varphi(y)=\varphi(x y)$ if $x, y, x y \in U$ implies that if $p(a)=p(b)$ for two elements $a, b \in U \times U$, then $\psi(a)=\psi(b)$. In other words, $\psi$ factors through the mapping $p$ and there exists a unique map $\varphi_{0}$ from $G$ to $H$ so that $\psi=\varphi_{0}{ }^{\circ}$ $\pi$. Weil's result says further that $\varphi_{0}$ is a homomorphism, and since $e \in U$, we see that $\varphi=\varphi_{0}$ almost everywhere; this shows the existence of $\varphi_{0}$.

Moreover $p$ is a Borel map and as $U \times U$ is standard it follows by wellknown results (see $[1$, Chapter I]) that $p$ induces a Borel isomorphism of the quotient space $(U \times U) / p$ with $G$ (as Borel spaces). Moreover since $\psi$ is a Borel map from $U \times U$ into $H$, it follows that $\psi$ viewed as a map from $(U \times U) / p$ into $H$ is Borel, but this is really just the map $\varphi_{0}$ once we identify $(U \times U) / p$ with G. Thus $\varphi_{0}$ is Borel and hence continuous (cf. Proposition 5).

Finally $\varphi_{0}$ is unique since if $\varphi_{1}$ were another such map, $\varphi_{1}=\varphi_{0}=\varphi$ say on the conull set $X \subset G$. But then necessarily $X \cdot X=G$ so $\varphi_{0}=\varphi_{1}$ everywhere.

This result clearly implies that $\underline{Z}^{1}(G, A)$ can be described as all continuous crossed homomorphisms and hence that $\underline{H}^{1}(G, A)$ consists of equivalence classes modulo the principal ones; that is, those of the form $g \rightarrow g \cdot a-a$. It follows 
immediately of course that $Z^{1}(G, A)$ consists of the continuous crossed homomorphisms also and so we obtain the following fact.

Corollary 1. The natural map $H^{1}(G, A) \rightarrow \underline{H}^{1}(G, A)$ is an isomorphism for all $A$.

The theory would not be complete if we did not take up some aspects of cohomology with coefficients in nonabelian coefficient groups, and it is known that that can be done at least in dimension one. Specifically let us define $Z^{1}(G, A)$ as Borel maps from $G$ into $A$ satisfying $f\left(s_{1} s_{2}\right)=s_{1} \cdot f\left(s_{2}\right) f\left(s_{1}\right)$; this family of functions is no longer a group but only a set. Moreover we say that $f$ and $f^{\prime}$ are equivalent if there exists $a \in A$ such that $f(s)=a f^{\prime}(s)(s \cdot a)^{-1}$. We define the quotient by this equivalence relation to be the set $H^{1}(G, A)$. Similarly, we define $Z^{1}(G, A)$ as the set of such $f$ satisfying the same identity almost everywhere, with $f \sim f^{\prime}$ if there is an $a$ with $f(s)=a f^{\prime}(s)(s \cdot a)^{-1}$ almost everywhere. Then $\underline{H}^{1}(G, A)$ is the quotient space. Again one has an obvious map $\varphi$ from $H^{1}(G, A)$ into $\underline{H}^{1}(G, A)$ and the same reasoning as above yields the following fact.

CoROllary 2. The natural map $H^{1}(G, A)$ to $\underline{H}^{1}(G, A)$ is an isomorphism of sets if $A$ is noncommutative.

We come to one of our major results concerning the cohomology groups.

TheOREM 4. We have $H^{n}(G, I(A))=\underline{H}^{n}(G, I(A))=(0)$ for $A \in P(G)$.

Consequently as a result of Theorem 2 we have

THEOREM 5. The canonical homomorphisms above $H^{n}(G, A) \rightarrow \underline{H}^{n}(G, A)$ are isomorphisms for all $n$ and all $A \in P(G)$.

The proof of Theorem 4 proceeds in several steps; the first shows that the functors $H^{n}$, and $\underline{H}^{n}$ satisfy a "Buchsbaum" criterion (cf. [7]).

Proposition 22. For any $A$, and for any $n>0$, the maps $H^{n}(G, A) \rightarrow$ $H^{n}(G, I(A))$ and $\underline{H}^{n}(G, A) \rightarrow \underline{H}^{n}(G, I(A))$ induced by the inclusion $A \rightarrow I(A)$ are the zero maps.

Proof. We note that

$$
\underline{C}^{n}(G, A)=U\left(G^{n}, A\right)=U\left(G^{n+1} \times G, A\right) \simeq U\left(G^{n-1}, U(G, A)\right)
$$

(by Fubini) and in turn this is $\underline{C}^{n-1}(G, I(A))$. We compose this isomorphism with the map of $\underline{C}^{n-1}(G, I(A))$ into itself given by

$$
F\left(g_{1}, g_{2}, \ldots, g_{n-1}\right)(x)=x^{-1} \cdot F\left(g_{1}, g_{2}, \ldots, g_{n-1}\right)\left(g_{n-1}^{-1}, \ldots, g_{1}^{-1} x\right) \text {. }
$$


All of this shows that for $f \in \underline{C}^{n}(G, A)$, the formula

$$
h\left(s_{1}, \ldots, s_{n-1}\right)(x)=x^{-1} \cdot h\left(s_{1}, \ldots, s_{n-1}, s_{n-1}^{-1} \ldots . s_{1}^{-1} x\right)
$$

makes good sense and defines an element $h \in \underline{C}^{n-1}(G, I(A))$. In exactly the same way we show that if $f \in C^{n}(G, A)$, then the same formula defines an element $h \in C^{n-1}(G, I(A))$.

We now assume that $f \in Z^{n}(G, A)$ (resp. $Z^{n}(G, A)$ ), and we compute the coboundary $\delta^{n-1}$ or $\delta^{n-1}$ of $f$ and we find that this is precisely equal to the image of $\pm f$ in $\underline{Z}^{n}(G, I(A))$ or $Z^{n}(G, I(A))$ obtained from the inclusion $A \rightarrow$ $I(A)$. We omit the routine details of the calculation.

REMARK. This is a special case of the usual argument for discrete groups to show that $H^{n}(G, I(A))=0$ where one takes $f \in Z^{n}(G, I(A))$ and defines

$$
h\left(g_{1}, \ldots, g_{n-1}\right)(x)=x^{-1} \cdot f\left(g_{1}, \ldots, g_{n-1}, g_{n-1}^{-1}, \ldots, g_{1}^{-1} x\right)(x) .
$$

In our case this is illegal as it would essentially involve taking a measurable function of $n+1$ variables and restricting it to an $n$-dimensional subset of its domain which is in fact a null set, and so this operation would be meaningless. We avoid this problem above by starting with an $f$ which is constant in one of its variables so that we can perform this construction in that case.

We now proceed with the second step in the proof of the theorem. Specifically let us assume that the theorem has been proved in all dimensions less than or equal to $n$ with $n \geqslant 1$, and let us prove it by induction for $n+1$. Let us consider the short exact sequence

$$
0 \rightarrow I(A) \rightarrow I(I(A)) \rightarrow U(I(A)) \rightarrow 0
$$

with corresponding long exact sequence

$$
H^{n}(G, I(I(A))) \rightarrow H^{n}(G, U(I(A))) \stackrel{g}{\rightarrow} H^{n+1}(G, I(A)) \underset{f}{\rightarrow} H^{n+1}(G, I(I(A))) .
$$

By the lemma above we see that $f$ is the zero map, and so by exactness, $g$ is surjective. On the other hand by Proposition 15, $U(I(A)) \simeq I(U(A))$, and hence by induction, $H^{n}(G, U(I(A)))=0$, and since $g$ is surjective, we immediately find $H^{n+1}(G, I(A))=0$, which completes our inductive step.

The final step which remains is to prove the theorem for $n=1$, and here we must compute directly.

Proposition 23. We have $H^{1}(G, I(A))=\underline{H}^{1}(G, I(A))=(0)$.

Proof. According to the corollary to Theorem 3, it is enough to do it for $H^{1}$. If $\bar{f} \in Z^{1}(G, I(A))$ we may by our Fubini theorem (Theorem 1 ) identify $\bar{f}$ with a Borel function $f$ on $G \times G$ into $A$ such that $f(s t, x)=f\left(t, s^{-1} x\right) \cdot f(s, x)$ holds for almost all triples $(s, t, x)$. Let use define $g(s, x)=f(x s, x)$ and then 


$$
g(s t, x)=f(x s t, x)=f\left(t, s^{-1}\right) \cdot f(x s, x)=g(s, x) \cdot f\left(t, s^{-1}\right)
$$

holds for almost all triples $(s, t, x)$. But now let $F(s)$ for $s \in G$ be the element of $I(A)$ given by $x \rightarrow g(s, x)$. Then of course $s \rightarrow F(s)$ is a Borel function of $G$ into $I(A)$. Further, let $F_{1}(s)$ be the image of $F(s)$ in $I(A) / A \simeq U(A)$ where $A$ is viewed as a trivial $G$-module so that $A \subset I(A)$ consists of constants. Then the equation above for $g$ says that $F(s t)=F(s) f\left(t, s^{-1}\right)$ or in terms of $F_{1}$, that $F_{1}(s t)=F_{1}(s)$ for almost all pairs $(s, t)$. It follows that $F_{1}$ is almost everywhere a constant in $U(A)$ and we pick an element $c \in I(A)$ projecting onto this constant so that now $F(s)=d(s) c$ for some Borel function $d$ from $G$ into $A$. Finally we see that $\bar{f}(x s)(x)=f(s, x)=d(s) c(x)$ for almost all pairs, and so by substitution of variables, we obtain $\bar{f}(s)(x)=d\left(x^{-1} s\right) c(x)$ and finally letting $a(x)=c(x)^{-1}$ and $b(s)=d\left(s^{-1}\right)$, we obtain $\bar{f}(s)(x)=b\left(s^{-1} x\right) a(x)^{-1}$ for almost all pairs, and by Fubini this holds for almost all $x$ for almost all $s$.

We view $a$ and $b$ as elements of $I(A)$ and then our equation reads $\bar{f}(g)=$ $(g \cdot b) a^{-1}$ for almost all $g$. On the other hand, Theorem 3 assures us that $\bar{f}$ as a function from $G$ to $I(A)$ is, in fact, continuous and since the other side of the above is patently continuous, we see that $\bar{f}(g)=(g \cdot b) a^{-1}$ holds for all $g$. Since $\bar{f}(e)=1$, we see that $b=a$ and so $\bar{f}(g)=(g \cdot b) b^{-1}=\underline{\delta}^{0}(b)$ is a coboundary. This completes the proof of the proposition and hence also the proofs of Theorems 4 and 5.

REMARK. The observant reader will recognize that this lemma for $A=T$, the circle group, is in fact for all intents and purposes Mackey's version of the Stone-von Neuman theorem [19] ; more specifically to obtain the full force of that result one would have to replace the circle group $T$ by the full unitary group $U$ on a separable Hilbert space $H$. This is a noncommutative group but we have defined cohomology sets in dimension one for noncommutative polonais $G$-modules. The point at issue then is simply the vanishing theorem $H^{1}(G, I(U))=(0)$ for unitary groups as above, but indeed the result is true for any noncommutative polonais group, and the same argument we give here extends immediately with no changes at all.

Corollary (of the proof of Proposition 23). We have $H^{1}(G, I(U)$ ) $=(0)$ for any polonais $U$, commutative or not, where the cohomology set is defined in the usual way as above as equivalence classes of continuous crossed homomorphisms.

We have now achieved our first goal-that is, the definition of rather naturally defined cohomology groups $H^{n}(G, A)$ for $A \in P(G)$ and we have in fact characterized them uniquely by a very simple set of axioms. Additionally, we have also shown that the two different definitions $H^{n}(G, A)$ and $\underline{H}^{n}(G, A)$ rather 
surprisingly coincide with each other, and consequently in what follows we will use these two notations interchangeably. We know what these groups are in dimension one and we will shortly show also that they give exactly what we want in dimension two.

Right now we establish one property of our groups immediately-namely the analogue of Shapiro's lemma about cohomology of induced modules. Recall our definition of induced modules $I_{H}^{G}(A)$ in $\S 3$. The following is a simply consequence of the circle of ideas above.

THEOREM 6. We have canonical isomorphisms $H^{n}(H, A) \simeq H^{n}\left(G, I_{H}^{G}(A)\right)$ for a closed subgroup $H$ of $G$ and $A \in P(H)$.

Proof. We view both sides as cohomological functors on $P(H)$ and observe the left-hand side, by definition, satisfies the axioms. Moreover, since $A \rightarrow$ $I_{H}^{G}(A)$ is a covariant functor preserving short exact sequences by Proposition 19, it follows that the right-hand side satisfies condition (1) of the definition. Moreover, $H^{0}\left(G, I_{H}^{G}(A)\right)=\left(I_{H}^{G}(A)\right)^{G} \cong A^{H}$ again by Proposition 19 so it also satisfies condition (2) of the definition. Finally, if $B=I_{(e)}^{H}(A)$, then $I_{H}^{G}\left(I_{(e)}^{H}(A)\right)=$ $I_{(e)}^{G}(A)$ by the proposition on inducing in stages so that $H^{n}\left(G, I_{H}^{G}\left(I_{(e)}^{H}(A)\right)\right)=(0)$ for $n>0$ and the right-hand side satisfies the third axiom. It follows from our uniqueness theorem that there are isomorphisms $H^{n}(H, A) \simeq H^{n}\left(G, I_{H}^{G}(A)\right)$ which are unique subject to extending the given isomorphism in dimension 0 .

We should note in this context that this theorem in dimension one, and for $A=T$, is essentially Mackey's imprimitivity theorem for induced representations [22]. To get the full force of that result one has to replace the $H$-module $A$ by a noncommutative module, namely the full unitary group on a separable Hilbert space, and as before one has cohomology sets. It is not hard to find a proof modeled on the ideas used here and we let the matter go with a statement.

Proposition 24 (MACKey IMPRIMITIVITY). If $U$ is any polonais H-module, commutative or not, and if $I_{H}^{G}(U)$ is the induced G-module defined as usual, we have an isomorphism of cohomology sets $H^{1}(H, U) \simeq H^{1}\left(G, I_{H}^{G}(U)\right)$.

5. We now want to examine the ramifications of the observation that the groups $\underline{H}^{n}(G, A)$ are not just abelian groups, but in fact are also topological groups. Since $\underline{C}^{n}(G, A)$ is polonais and $\underline{\delta}^{n}$ is continuous, $\underline{Z}^{n}(G, A)$ is polonais and $\underline{B}^{n}(G, A)$ is the continuous image of a polonais group, although it may not be a closed subgroup. In any case, the quotient $\underline{H}^{n}(G, A)$ is a (not necessarily Hausdorff) topological group. It is Hausdorff if and only if $\underline{B}^{n}(G, A)$ is closed, and in this case it is a polonais group. Moreover if $(\bar{e})$ denotes the closure of the identity in $\underline{H}^{n}(G, A)$ so that it is $\underline{B}^{n}(G, A) / \underline{B}^{n}(G, A)$, then $H^{n}(G, A) /(\bar{e})$ is always a polonais group. We want to show now that the $\underline{H}^{n}(G, A)$ are in fact functors 
into the category of topological groups. We first note the following facts

Proposition 25. (1) If $f$ is a continuous G-homomorphism from $A$ to $B$, the induced map $\underline{H}^{n}(G, A) \rightarrow H^{n}(G, B)$ is continuous.

(2) If $0 \rightarrow A^{\prime} \rightarrow A \rightarrow A^{\prime \prime} \rightarrow 0$ is short exact, then the coboundary maps $\partial^{n}: \underline{H}^{n}\left(G, A^{\prime \prime}\right) \rightarrow \underline{H}^{n+1}\left(G, A^{\prime}\right)$ are continuous.

Proof. For (1) observe that the induced map on cochains is continuous by results in $\$ 2$ and hence so is the map on cohomology. On the other hand, part (2) is somewhat more subtle. We first select a Borel cross section $c: A^{\prime \prime} \rightarrow$ $A$, and note that if $f \in \underline{Z}^{n}\left(G, A^{\prime \prime}\right)$, then $c \circ f \in \underline{C}^{n}(G, A)$ and according to the corollary to Proposition $8, f \rightarrow c \circ f$ is a Borel map. Finally $\underline{\delta}^{n}(c \circ f) \in$ $\underline{Z}^{n+1}\left(G, A^{\prime}\right)$ and its class is, by definition, the image of the class of $f$ under $\partial^{n}$. Since $\underline{\delta}^{n}$ is continuous $f \rightarrow \underline{\delta}^{n}(c \circ f)$ is a Borel map. We are interested in the continuity of the map $j\left(\delta^{n}(c \circ f)\right)$ where $j$ is the projection of $\underline{Z}^{n}\left(G, A^{\prime}\right)$ onto $\underline{H}^{n}\left(G, A^{\prime}\right)$. Now this group may not be Hausdorff but we claim that a map into such a group is continuous if and only if this map composed with the quotient map onto $\underline{H}^{n}\left(G, A^{\prime}\right)$ modulo the closure of the identity element is continuous. But now this is the same as considering the map $\phi(f)=p\left(\delta^{n}(c \circ f)\right)$ where $p$ is the map of $\underline{Z}^{n}\left(G, A^{\prime}\right)$ onto $\underline{Z}^{n}\left(G, A^{\prime}\right) / \underline{B}^{n}(G, A)$. Now indeed this map $\phi$ is Borel as $p$ is continuous, and it follows by a standard theorem (cf. Proposition 5) that $\phi$ is then continuous, and we are done.

It is helpful if we have a certain strengthening of this in a special case. Recall that Theorem 2 gives rise to isomorphisms $\underline{H}^{n}(G, U(A)) \simeq \underline{H}^{n+1}(G, A)$ for $n \geqslant 1$ and where $U(A) \simeq I(A) / A$.

Proposition 26. The isomorphisms $\underline{H}^{n}(G, U(A)) \simeq \underline{H}^{n+1}(G, A)$ are homeomorphisms.

ProOF. We have to construct a continuous inverse for $\partial^{n}$, and indeed if $f \in \underline{Z}^{n+1}(G, A)$ we defined

$$
\phi_{1}(f)\left(s_{1}, \ldots, s_{n}\right)(x)=x^{-1} \cdot f\left(s_{1}, ., s_{n}, s_{n}^{-1} \cdot \ldots \cdot s_{1}^{-1} x\right) \in \underline{C}^{n}(G, I(A)) .
$$

Then by using Proposition 6, we see that $f \rightarrow \phi_{1}(f)$ is continuous and if $q$ is the projection of $\underline{C}^{n}(G, I(A))$ onto $\underline{C}^{n}(G, U(A))$, then $f \rightarrow \phi(f)=q\left(\phi_{1}(f)\right)$ is continuous, and we observe in fact that $\phi(f) \in \underline{Z}^{n}(G, U(A))$. The induced map on cohomology $\underline{H}^{n+1}(G, A) \rightarrow \underline{H}^{n}(G, U(A))$ is therefore continuous and is precisely the inverse of $\partial^{n}$ and the proof is complete.

We have the following corollary which follows by iteration.

COROLlaRY. The group isomorphisms $\underline{H}^{n}(G, A) \simeq \underline{H}^{1}\left(G, U_{n-1}(A)\right)$ as defined in the corollary to Theorem 2 are homeomorphisms. 
The above gives a description both algebraically and topologically of the higher groups in terms of $H^{1}$ and indeed one could if desired go down to the groups $H^{0}$. We know the algebraic structure of $H^{1}(G, B)$, namely the classes of continuous crossed homomorphisms of $G$ into $B$. This group also has a rather directly and naturally defined topology for we can give $Z^{1}(G, B)$ the compact open topology as functions on $G$. (One could give a similar description of this topology over on $\underline{Z}^{1}(G, B)$, but it gets a bit involved as we are dealing there with equivalence classes of functions.) In any case let us give

$$
H^{1}(G, B)=Z^{1}(G, B) / B^{1}(G, B)
$$

the quotient topology. We can do exactly the same for $B$ noncommutative where we have cohomology sets rather than groups. The following answers the obvious question about these two topologies.

THEOREM 7. The isomorphism $H^{1}(G, B) \simeq \underline{H}^{1}(G, B)$ is a homeomorphism from the "compact open" topology on the first group, or set, if $B$ is nonabelian, to the topology on $\underline{H}^{1}(G, B)$ coming from $\underline{C}^{1}(G, B)=U(G, B)$.

Proof. We shall prove that the corresponding map $Z^{1}(G, B) \simeq \underline{Z}^{1}(G, B)$ is a homeomorphism and that will suffice. We note indeed that the map is an algebraic isomorphism by Theorem 4 and the observation that if $f_{i} \in Z^{1}(G, B)$ and agree a.e., then $f_{1}=f_{2}$. Now suppose that $f_{n} \rightarrow f$ in $Z^{1}(G, B)$ in the compact open topology; then $f_{n} \rightarrow f$ pointwise and hence surely in the topology of $\underline{Z}^{1}(G, B)$ by Proposition 6 . If $B$ is abelian we can argue that $Z^{1}(G, B)$ is easily seen to be polonais, and since we know $\underline{Z}^{1}(G, B)$ is, it follows that the map above is a homeomorphism by the closed graph theorem (cf. Proposition 5). (Note that this will work if we know only, say, that $Z^{1}(G, B)$ is an analytic Borel space; the fact that it is polonais would follow from this and the above argument.) On the other hand, if $B$ is not abelian, we must proceed differently and more directly. What is at issue is the fact that if $f_{n}$ is a sequence of crossed homomorphisms of $G$ to $B$ converging to $f$ in measure, then $f_{n} \rightarrow f$ uniformly on compact sets. First of all, for simplicity we may use the device employed in Theorem 3 and replace all crossed homomorphisms by ordinary homomorphisms, which we do.

Now as $f_{n} \rightarrow f$ in $\underline{Z}^{1}(G, B)$ every subsequence $g_{m}$ of the $f_{n}$ has a subsequence $h_{k}$ which converges pointwise almost everywhere to $f$, say $h_{k}(s) \rightarrow f(s)$ for $s \in K$, a conull set. But as $K \cdot K=G$ and $h_{k}(s t)=h_{k}(s) h_{k}(t) \rightarrow f(s) f(t)=$ $f(s t)$, it follows that $h_{k} \rightarrow f$ everywhere. It follows then by standard reasoning that the original sequence $f_{n}$ converges to $f$ pointwise everywhere. Now the problem is to make this uniform. Let $\rho$ be a bounded left invariant metric on $A$, and let $E$ be any compact set of positive finite Haar measure on $G$ with $E=E^{-1}$ 
Then given any subsequence $g_{m}$ of the $f_{n}$ and given any $\mu(E)>\delta>0$, we can find by an obvious variant of Egorov's theorem a subsequence $h_{k}$ of $g_{m}$ and a set $F \subset E$ with $\mu(E-F)<\delta$ and with $h_{k}(s) \rightarrow f(s)$ uniformly with respect to $\rho$ for $s \in F$. We can also select $F=F^{-1}$ so that $F \cdot F$ contains a neighborhood of the identity, say $U$ as $\mu(F)>0$. Let $\epsilon>0$, and choose $N$ so large that if $n>N$, we have $\rho\left(h_{n}(t), f(t)\right)<\epsilon / 2$ uniformly for $t \in F$. Moreover, since $E$ is compact, $f(E) \subset B$ is compact, and it follows that we may find a $\delta$ sufficiently small such that if $\rho(b, e)<\delta$ then $\rho\left(s^{-1} b s, e\right)<\epsilon / 2$ for every $s \in f(E)$; in particular, $\rho\left(b_{1} f(t), b_{2} f(t)\right)<\epsilon / 2$ for $t \in F$ whenever $\rho\left(b_{1}, b_{2}\right)=\rho\left(b_{2}^{-1} b_{1}, e\right)<\delta$. Then we make our $N$ above larger, if necessary, so that in addition, $\rho\left(h_{n}(s), f(s)\right)<\delta$ for $n>N$ uniformly for $s \in F$. Now if $u \in U$ is arbitrary, $u=s t$, with $s, t \in F$ and

$$
\begin{aligned}
\rho\left(h_{n}(u), f(u)\right) & =\rho\left(h_{n}(s t), f(s t)\right) \\
& \leqslant \rho\left(h_{n}(s) h_{n}(t), h_{n}(s) f(t)\right)+\rho\left(h_{n}(s) f(t), f(s) f(t)\right) \\
& =\rho\left(h_{n}(t), h(t)\right)+\rho\left(h_{n}(s) f(t), f(s) f(t)\right) \\
& <\epsilon / 2+\epsilon / 2=\epsilon \text { for } n>N .
\end{aligned}
$$

Thus $h_{n} \rightarrow f$ uniformly on $U$ and hence, by an easy argument, on any left translate of $U$, and hence on any compact set in $G$. Thus we have shown that any subsequence of the original sequence $f_{n}$ has a subsequence converging uniformly on compact sets to $f$ which is enough to show that $f_{n}$ itself converges uniformly on compact sets to $f$ and this completes the proof.

In the course of the argument we have, of course, shown the following fact of some interest which we cannot point to in the literature in this generality. Many special cases are, of course, well known and classical.

THEOREM 8. If $f_{n}$ is a sequence of homomorphisms (or crossed homomorphisms) of a locally compact separable group $G$ into a separable metric group $B$ which converge pointwise, then they converge uniformly on compact sets.

These results give a rather direct and natural description of the topology on $\underline{H}^{1}(G, B)$ and, hence, using the corollary of Proposition 26, we get some sort of description of the topology on $\underline{H}^{n}(G, A)$ for any $n$ and any $A$.

In the above we have concentrated on the behavior of the topology on $H^{n}(G, A)$ as $A$ varies, but it is equally important to investigate the behavior as $G$ varies. Specifically, if $\varphi: H \rightarrow G$ is a continuous homomorphism and if $A \in P(H)$ and $A \in P(G)$ with $\varphi(h) \cdot a=h \cdot a$, where the two dots refer to the actions of $G$ and $H$ on $A$ and $A$, respectively, we expect induced maps $\left(\varphi_{*} f\right): \underline{H}^{n}(G, A) \rightarrow$ $\underline{H}^{n}(H, A)$ which should be continuous. One usually defines these maps by associated cochain maps 


$$
\left(\phi_{*} f\right)\left(s_{1}, \ldots, s_{n}\right)=f\left(\varphi\left(s_{1}\right), \ldots, \varphi\left(s_{n}\right)\right)
$$

which is fine for $f \in C^{n}(G, A)$ and gives the desired homomorphisms from $H^{n}(G, A)$ to $H^{n}(H, A)$. However, if we tried the same thing for the groups $\underline{H}^{n}(\cdot, B)$, we run into trouble, for suppose $\varphi(H) \subset G$ is a Haar null set (e.g. $\bar{Z} \subset R$ ). Then the right-hand side of (*) above is meaningless since we are attempting to evaluate an equivalence class of measurable functions on a null set. Indeed the very existence of such maps $\underline{H}^{n}(G, A) \rightarrow \underline{H}^{n}(H, A)$, much less their continuity, would be somewhat mysterious if taken in isolation.

Proposition 27. The maps $\left(\varphi_{*} f\right): \underline{H}^{n}(G, A) \rightarrow \underline{H}^{n}(H, A)$ are continuous.

Proof. We proceed by induction on $n$, the result being clear for $n=0$. For $n=1$, it is enough to consider the cocycle groups $Z^{1}$ which we identify as groups of continuous crossed homomorphisms with the compact open topology by Theorem 7. The continuity for the map $Z^{1}(G, A) \rightarrow Z^{1}(H, A)$ in question is then clear, since $\varphi$ maps compact sets in $H$ into compact sets in $G$.

Assume now that we have established the lemma in dimension $n \geqslant 1$; we prove it in dimension $n+1$. We write the usual exact sequence $0 \rightarrow A \rightarrow I(A)$ $\rightarrow U(A) \rightarrow 0$ of $G$-modules, and use the map of $H$ into $G$ to make this sequence into a sequence of $H$ modules in a compatible way. The long exact sequences of cohomology then read:

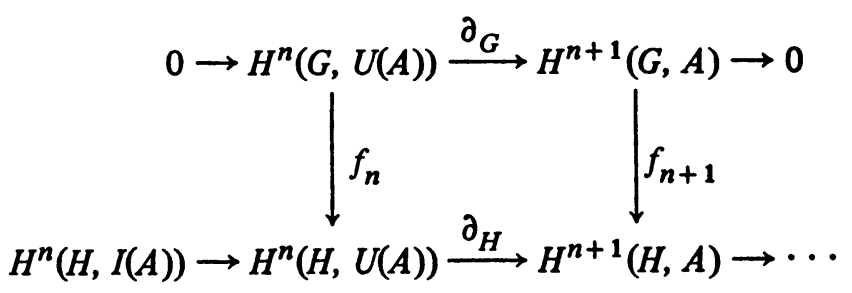

We know that $f_{n}$ is continuous and that $\partial_{H}$ is continuous. Moreover since $\partial_{G}$ is a homeomorphism by Proposition 26, $f_{n+1}=\partial_{H} f_{n} \partial_{G}^{-1}$ is continuous as desired.

We conclude this section with a description of the situation regarding the spectral sequence of a group extension in our context-a tool that has been shown to be absolutely invaluable. Let $H$ be a closed normal subgroup of $G$ and let $A$ be a $G$-module. Then one hopes for a spectral sequence $E_{r}^{p, q}$ converging to $H^{*}(G, A)$ with $E_{2}^{p, q} \simeq H^{p}\left(G / H, H^{q}(H, A)\right)$. One should consult [16] for the algebraic treatment and [29] for a first approximation of a topological treatment. For this statement to begin to make sense we need a topology on the groups $H^{q}(H, A)$, and fortunately we have one rather naturally defined via $\underline{H}^{q}(H, A)$ although it may unfortunately fail to be Hausdorff. In any case we have the following fact. 
Proposition 28. The group $\underline{H}^{q}(H, A)$ is a topological $G / H$ module and hence if it is Hausdorff, it is in $P(G / H)$.

Proof. We note that the group $G$ acts on the polonais group $\underline{Z}^{q}(H, A)$ by $(s \cdot f)\left(h_{1}, \ldots, h_{q}\right)=s \cdot f\left(s h_{1} s^{-1}, \ldots, s h_{q} s^{-1}\right)$. The function $s \cdot f$ is continuous in $s$ by an easy variant to Proposition 12, and is continuous in $f$ by Proposition 6. Hence the action is jointly continuous by Proposition 11. By going over to the quotient $\bmod \underline{B}^{q}(H, A)$, we see that $\underline{H}^{q}(H, A)$ is indeed a topological $G$ module.

THEOREM 9. There is a spectral sequence $E_{r}^{p, q}$, in fact the same one as in Theorem 1.1 of [29] if $A$ is locally compact, converging to $\underline{H}^{*}(G, A)$ such that $E_{2}^{p, q}=\underline{H}^{p}\left(G / H, \underline{H}^{q}(H, A)\right)$ at least whenever $\underline{H}^{q}(H, A)$ is Hausdorff.

Proof. The argument in [29] works virtually verbatim and indeed the situation is even simpler since we have available our Fubini theorem, Theorem 1, and the proposition above. In [29] we introduced the notion of the complex $C^{*}(H, A)$ being regular in dimension $i$. What is needed here is that $C^{*}(H, A)$ is regular in the sense that we can find a Borel mapping $\phi_{i}$ from $\underline{B}^{i}(H, A)$ into $\underline{C}^{i-1}(H, A)$ which is a right inverse for the coboundary differential. Such a Borel map will have all the necessary properties as described in Definition 1.5 of [29]. In our context it is easy to construct $\phi_{i}$ for we simply take a Borel cross section $s_{i}$ from $D^{i}=\underline{C}^{i-1}(H, A) / \underline{Z}^{i-1}(H, A)$ into $\underline{C}^{i-1}(H, A)$. Then $\underline{\delta}^{i-1}$ induces an injection $d^{i}$ of the polonais group $D^{i}$ onto $\underline{B}^{i}(H, A) \subset \underline{C}^{i}(H, A)$. Although $d^{i}$ is not a homeomorphism unless $\underline{B}^{i}(H, A)$ is closed it is a Borel isomorphism (cf. Proposition of Chapter I of [1]). We then take $\phi_{i}=s_{i} \circ d_{i}^{-1}$.

REMARK. Since one might think of $\underline{H}^{q}(H, A)$ as a pseudo-polonais $G / H$ module, one might attempt to construct its cohomology groups, since we do have a definition of what it means for a function from a space $X$ into such groups to be Borel. This definition is fortuitous in that it is precisely what comes up when one looks closely at the spectral sequence. One thus could in principal identify all the terms of the spectral sequence in terms of such suitably defined cohomology groups. We shall, however, refrain from pursuing this approach and content ourselves with the description in case $\underline{H}^{q}(H, A)$ is Hausdorff.

6. There is one essential element of our development which is missing so far and that is the identification of $H^{2}(G, A)$ as a set of group extensions. We now proceed to fill this gap; it is interesting to note that our argument here, which is valid for general polonais $A$, is in some ways simpler and more constructive than the original argument of Mackey [24] in the case $A$ locally compact. The simplification results from an effective use of the idea of identifying cocycles equal almost everywhere. 
THEOREM 10. We have an isomorphism $H^{2}(G, A) \simeq \underline{H}^{2}(G, A) \simeq \operatorname{Ext}(G, A)$, the group of all equivalence classes of topological group extensions of $G$ by the $G$-module $A$.

Proof. First let us suppose that $1 \rightarrow A \stackrel{i}{\rightarrow} E \stackrel{\pi}{\rightarrow} G \rightarrow 1$ is such an extension. It follows from Proposition 3 that $E$ is polonais, and then by the fundamental cross section theorem (cf. Proposition 4), there exists a Borel map $s$ from $G$ to $E$ with $(\pi \circ s)(g)=g$. Then we define $a(g, h)=i^{-1}\left(s(g) s(s) s(g h)^{-1}\right)$ and note that it is an element of $Z^{2}(G, A)$ with cohomology class $\alpha(E)$ independent of the choice of $s$ which provides a map of $\operatorname{Ext}(G, A)$ into $H^{2}(G, A)$. It is purely algebraic and classical that $\alpha$ is a group homomorphism (using the Baer product on $\operatorname{Ext}(G, A)$ ). We show first that $\alpha$ is injective. If $\alpha(E)=0$, then we claim that we can choose the Borel cross section $s$ so that the corresponding cocycle $a(g, h)$ $=1$. This says that $s$ is a homomorphism, and being Borel it is automatically continuous. Then the map $(a, g) \rightarrow i(a) s(g)$ defines a continuous bijective homomorphism of the semidirect product $A \cdot B$ onto $E$ as group extensions. Since $A \cdot G$ and $E$ are polonais, this map is a homeomorphism and $E$ is the identity element of $\operatorname{Ext}(G, A)$. It remains to show that $\alpha$ is surjective, which of course is the hard part.

For this let $\underline{b} \in H^{2}(G, A)$ and let $b \in Z^{2}(G, A)$ be a representative. By way of motivation for what follows, let us note that the image of $\underline{b}$ in $H^{2}(G, I(A))$ under the map induced by the inclusion $A \rightarrow I(A)$ is the trivial class, and hence the corresponding group extension is the semidirect product $I(A) \cdot G$. It is then very natural to expect that the group extension of $G$ by $A$ which we are seeking as a preimage for the class $\underline{b}$ should be constructible as a specific subgroup of $I(A) \cdot G$, and this is precisely what we shall do.

We note that for fixed $g$ the function $x \rightarrow x^{-1} \cdot b\left(g, g^{-1} x\right)$ can be viewed as an element of $I(A)$ and as such we denote it by $T(g)$; then define the element $L(g)$ of $I(A) \cdot G$ as the pair $L(g)=(T(g), g)$. We compute that $L(g) L(h)=$ $(A(g, h), g h)$ where $A(g, h)$ as a function of $x$ is given by

Now

$$
A(g, h)(x)=x^{-1} \cdot\left(b\left(g, g^{-1} x\right)+g \cdot b\left(h, h^{-1} g^{-1} x\right)\right)
$$

$$
L(g h)=(t(g h), g h) \text { and } T(g h)(x)=x^{-1} \cdot\left(b\left(g h, h^{-1} g^{-1} x\right)\right)
$$

An elementary application of the cocycle identity satisfied by $b$ shows that

$$
A(g, h)(x)=T(g h)(x)+x^{-1} \cdot(b(g, h)) \quad \text { or } \quad A(g, h)=T(g h)+i(b(g, h))
$$

where $i$ is the map embedding $A$ into $I(A)$. Thus we see that $L(g) L(h)=$ $i(b(f, g), e) \cdot L(g h)$ where $e$ is the identity of $G$. Let us consider the subgroup $E$ of the semidirect product $I(A) \cdot G$ generated by the elements $L(g), g \in G$, and 
$i(a), a \in A$. The above identity for $L(g) L(h)$ shows that every element of $E$ can be written as $i(a) L(g)$, and indeed this representation is unique, for if $i\left(a^{\prime}\right) L\left(g^{\prime}\right)=$ $i(a) L(g)$, then $\left(x^{-1} \cdot a^{\prime}+x^{-1} \cdot b\left(g^{\prime}, g^{\prime-1} x\right), g^{\prime}\right)=\left(x^{-1} \cdot a+x^{-1} \cdot b\left(g, g^{-1} x\right)\right.$, g) so $g=g^{\prime}$ and $a-a^{\prime}=b\left(g, g^{-1} x\right)-b\left(g, g^{-1} x\right)=0$ a.e. so $a=a^{\prime}$. We give $E$ the topology it inherits as a subgroup of $I(A) \cdot G$, and we show that it is a closed subset, hence polonais.

Let $p$ be the projection of $I(A)$ onto $U(A)$; then the equation for $L(g) L(h)$ says that $p \circ L$ is a homomorphism of $G$ into $U(A)$. It is clear moreover that $L$ is a Borel function into $I(A)$ by use of Proposition 8, and hence $p \circ L$ is Borel, and also cuntinuous. Thus if $g_{n} \rightarrow g$ in $G, p \circ L\left(g_{n}\right) \rightarrow p \circ L(g)$ in $U(A)$, and hence there is a sequence $c_{n}$ in $A$ such that $i\left(c_{n}\right)+L\left(q_{n}\right) \rightarrow L(g)$. Now suppose we have a sequence $i\left(a_{n}\right) L\left(g_{n}\right)$ in $E$ converging to an element $(c, g)$ of $I(A) \cdot G$. Then of course $g_{n} \rightarrow g$ and $i\left(a_{n}\right)+L\left(g_{n}\right) \rightarrow c$ and, by the above, $i\left(a_{n}\right)-i\left(c_{n}\right)$ $+L(g) \rightarrow c$ and, hence, the difference $a_{n}-c_{n}$ must converge to some limit $d$, and we have $d+L(g)=c$ so that $(c, g)=i(d) L(g)$ is in $E$ and $E$ is closed.

Further $i$ gives a topological embedding of $A$ into $E$ as a closed normal subgroup. Moreover $\pi(i(a) L(g))=g$ defines a homomorphism of $E$ onto $G$ with kernel $i(A)$. The map $\pi$ is continuous as it is the restriction to $E$ of the natural projection of $I(A) \cdot G$ onto $G$. As $E$ is polonais, $E$ is then an extension of $A$, and as the action is correct, of the $G$ module $A$. Moreover $E$ comes equipped with a Borel section $g \rightarrow L(g)$ of $G$ in $E$ such that the associated cocycle is precisely $b$. Thus $E$ is an extension of $G$ by $A$ corresponding to given cohomology class $\underline{b}$, and the proof is complete.

We defer a discussion in more detail of the topology on $\underline{H}^{2}(G, A)$ until a following paper where it fits more naturally.

We might remark that one may show just as in [1, Chapter IV], that $H^{3}(G, A)$ contains the naturally defined obstructions arising in the consideration of nonabelian extensions. It is a routine exercise which we shall not pursue further.

7. The above completes the general description and properties of the cohomology groups $H^{n}(G, A)$ and $\underline{H}^{n}(G, A)$. In conclusion we should point out the relation of these groups with other constructions. D. Wigner's thesis [34] clarifies these relationships and let us recall his major results here. If $G$ is any topological group, it has a classifying space $B_{G}$ [27], and if $A$ is any $G$-module with the discrete topology, one may construct a locally constant sheaf $\underline{A}$ on $B_{G}$ with fiber $A$ with the nonconstancy reflecting the action of $G$ on $A$. Then Wigner shows that:

(1) If $G$ is finite dimensional, and $A$ is discrete, there are isomorphisms $H^{n}(G, A) \simeq H^{n}\left(G_{B}, \underline{A}\right)$ (sheaf cohomology). 
Note that this could not be expected to hold for modules $A$ with a topology since the topology on $A$ enters on the left-hand side of the above but cannot enter on the right. We note, in particular, that this result says that our groups coincide with the groups used in class field theory $H^{n}(G, A)$ defined for profinite groups $G$ and discrete modules $A$ [10]. Wigner also shows:

(2) If $G$ is finite dimensional and $V$ a finite dimensional vector space which is a linear $G$-module, then $H^{n}(G, V) \simeq H_{c}^{n}(G, V)$ where the latter group is the one defined by Hochschild and Mostow [15], [28] using continuous cochains rather than Borel cochains.

On the other hand, our groups do not coincide with the groups introduced by Johnson [14] for modules which are Banach spaces. Note that he restricts attention only to bounded cochains.

It would appear on the basis of the axioms characterizing our groups $H^{n}(G, A)$ or $\underline{H}^{n}(G, A)$, and on the interpretation of these groups in dimensions one and two, and on the basis of the isomorphisms above $m$ special cases, that our groups $H^{n}(G, A)$ have a certain claim to being the appropriate generalization of the Eilenberg-Mac Lane groups. In a subsequent paper we shall indicate a number of diverse applications of these groups to such topics as the structure of topological groups.

\section{REFERENCES}

1. L. Auslander and C. C. Moore, Unitary representations of solvable Lie groups, Mem. Amer. Math. Soc. No. 62 (1966). MR 34 \#7723.

2. N. Bourbaki, Éléments de mathématique. VIII. Topologie générale. Chap. 9, Actualités Sci. Indust., no. 1045, Hermann, Paris, 1948. MR 10, 260.

3. S. Banach, Théorie des opérations linéaires, Monografie Mat., tom 1, PWN, Warsaw, 1932; reprint, Chelsea, New York, 1955. MR 17, 175.

4. L. G. Brown, Extensions of topological groups, Pacific J. Math. 39 (1971), 71 78. MR 46 \#6384.

5. $\longrightarrow$ Topologically complete groups, Proc. Amer. Math. Soc. 35 (1972), 593600. MR 46 \#7435.

6. Completeness, separability, metrizability and extensions of topological groups (in preparation).

7. D. Buchsbaum, Satellites and universal functors, Ann. of Math. (2) 71 (1960), 199-209. MR 22 \#3751.

8. J. Dixmier, Les algèbres d'opérateurs dans l'espace Hilbertien (Algèbres de von Neumann), Gauthier-Villars, Paris, 1957. MR 20 \#1234.

9. - Dual et quasi-dual d'une algèbre de Banach involutive, Trans. Amer. Math. Soc. 104 (1963), 273-283. MR 25 \#3384.

10. A. Douady, Cohomologies des groupes compacts totalement discontinues, Séminaire Bourbaki 12e année: 1959/60, Exposé 189, Secrétariat mathématique, Paris, 1960. MR 23 \#A2273.

11. N. Dunford and J. T. Schwartz, Linear operators. I: General theory, Pure and Appl. Math., vol. 7, Interscience, New York, 1958. MR 22 \#8302.

12. W. T. van Est, Group cohomology and Lie algebra cohomology in Lie groups. I, II, Nederl. Akad. Wetensch. Proc. Ser. A 56 = Indag. Math. 15 (1953), 484-504. MR 15, 505.

13. A. Guichardet, Sur la cohomologie des groupes topologique. I, II, Bull. Sci. Math. 
(2) 95 (1971), 161-176; ibid. (2) 96 (1972), 305-322. MR 46 \#6385; 49 \#5219. (1972).

14. B. E. Johnson, Cohomology in Banach algebras, Mem. Amer. Math. Soc. No. 127

15. G. P. Hochschild and G. D. Mostow, Cohomology of Lie groups, Illinois J. Math. 6 (1962), 367-401. MR 26 \#5092.

16. G. P. Hochschild and J.-P. Serre, Cohomology of group extensions, Trans. Amer. Math. Soc. 74 (1953), 110-134. MR 14, 619. 1136.

17. J. L. Kelley, General topology, Van Nostrand, Princeton, N. J., 1955. MR 16,

18. C. Kuratowski, Topologie. Vol. I, 3rd ed., PWN, Warsaw, 1952. MR 14, 1000.

19. G. W. Mackey, A theorem of Stone and von Neumann, Duke Math. J. 16 (1949), 313-326. MR 11, 10.

20. - Induced representations of locally compact groups. I, Ann. of Math. (2)

55 (1952), 101-139. MR 13, 434.

21. Induced representations of locally compact groups. II. The Frobenius reciprocity theorem, Ann. of Math. (2) 58 (1953), 193-221. MR 15, 101.

22. Imprimitivity for representations of locally compact groups. I, Proc. Nat. Acad. Sci. U. S. A. 35 (1949), 537-545. MR 11, 158.

23. Les ensembles borélien et les extensions des groupes, J. Math. Pures Appl. (9) 36 (1957), 171-178. MR 19, 752.

24. Borel structure in groups and their duals, Trans. Amer. Math. Soc. 85 (1957), 134-165. MR 19, 752.

25. Point realizations of transformation groups, Illinois J. Math. 6 (1962), 327-335. MR 26 \#1424.

26. L. Michel, Sur les extensiones centrales du groupe de Lorentz inhomogene connexe, Nuclear Phys. 57 (1965), 356-385. MR 35 \#1699.

27. J. Milnor, Construction of universal bundles. II, Ann. of Math. (2) 63 (1956), 430-436. MR 17, 1120.

28. G. D. Mostow, Cohomology of topological groups and solvmanifolds, Ann. of Math. (2) 73 (1961), 20-48. MR 23 \#A2484.

29. C. C. Moore, Extensions and low dimensional cohomology theory of locally compact groups. I, Trans. Amer. Math. Soc. 113 (1964), 40-63. MR 30 \#2106.

30. Extensions and low dimensional cohomology theory of locally compact groups. II, Trans. Amer. Math. Soc. 113 (1964), 64-86. MR 30 \#2106.

31. - Restrictions of unitary representations to subgroups and ergodic theory: Group extensions and group cohomology, Group Representations in Math. and Phys. (Battelle Seattle 1969 Rencontres), Lecture Notes in Phys., vol. 6, Springer, Berlin, 1970, pp. 1-35. MR 43 \#4955.

32. Group extensions of p-adic and adelic linear groups, Inst. Hautes Études Sci. Publ. Math. No. 35 (1968), 157-222. MR 39 \#5575.

33. A. Weil, Sur certains groupes d' opérateurs unitaires, Acta Math. 111 (1964), 143-211. MR 29 \#2324.

34. D. Wigner, Algebraic cohomology of topological groups, Trans. Amer. Math. Soc. 178 (1973), 83-93.

DEPARTMENT OF MATHEMATICS, UNIVERSITY OF CALIFORNIA, BERKELEY, CALIFORNIA 94720 